\title{
Intra- and inter-shell Kondo effects in carbon nanotube quantum dots
}

\author{
Damian Krychowski and Stanisław Lipiński ${ }^{\mathrm{a}}$ \\ Institute of Molecular Physics Polish Academy of Sciences, M. Smoluchowskiego 17, 60-179 Poznań, Poland
}

Received 28 September 2017

Published online 15 January 2018

(C) The Author(s) 2018. This article is published with open access at Springerlink.com

\begin{abstract}
The linear response transport properties of carbon nanotube quantum dot in the strongly correlated regime are discussed. The finite-U mean field slave boson approach is used to study many-body effects. Magnetic field can rebuilt Kondo correlations, which are destroyed by the effect of spin-orbit interaction or valley mixing. Apart from the field induced revivals of $\mathrm{SU}(2)$ Kondo effects of different types: spin, valley or spin-valley, also more exotic phenomena appear, such as SU(3) Kondo effect. Threefold degeneracy occurs due to the effective intervalley exchange induced by short-range part of Coulomb interaction or due to the intershell mixing. In narrow gap nanotubes the full spin-orbital degeneracy might be recovered in the absence of magnetic field opening the condition for a formation of SU(4) Kondo resonance.
\end{abstract}

\section{Introduction}

In the last two decades carbon nanotubes (CNTs) have attracted tremendous interest from fundamental science and technological perspectives [1-6]. The unique electronic properties of these thin, hollow cylinders formed from graphene are due to confinement of electrons normal to the nanotube axis. In a very crude approach (zone-folding approximation $[7,8]$ ), the bandstructure of nanotube is obtained from the bandstructure of graphene by imposing periodic boundary conditions along the circumference. Depending on the way graphene is rolled up, carbon nanotube can be either metallic or semiconducting. In this simple picture bandgap depends on the minimum separation of the quantization lines from Dirac points and is inversely proportional to diameter. The zone folding approximation breaks down for tubes of small diameter. In this case the curvature induced corrections to the overlaps between adjacent orbitals cannot be neglected. In this way one can understand experimentally observed opening of a narrow gap $(\sim 10 \mathrm{meV})$ even in nominally metallic tubes (these systems are sometimes called nearly metallic carbon nanotubes [9]). Similar effect occurs also due to the strain. The resulting gaps are inversely proportional to the square of diameter, depend on chiral angles and are typically much smaller than the gaps resulting from circular quantization. The dispersion laws of narrow gap nanotubes differ considerably from the dispersions of wide gap nanotubes and consequently distinctly different is their behavior in magnetic fields [9]. Considerations of the present work are addressed to semiconducting and narrow gap carbon nanotubes. We focus on strong

\footnotetext{
${ }^{\mathrm{a}}$ e-mail: lipinski@ifmpan.poznan.pl
}

correlation effects, which are of importance in quantum dots (CNTQDs) formed from a short part of nanotube e.g. by introducing a confining potential in the longitudinal direction. Electron correlations in CNTQDs are strong due to the one-dimensional nature of confinement and due to the low dielectric constant especially in suspended nanotubes [10]. As the size of the dot decreases the charging energy of a single excess charge on the dot increases. Transmission of the contacts determines the regime of charge transport. For very weak transparency charging effects dominate transport at low temperature and the electrons enter the dot one by one yielding the well known Coulomb blockade oscillations of conductance as a function of the gate voltage. For more open contacts the role of higher order tunneling processes (cotunneling) increases what results at low temperatures in formation of many-body resonances at the Fermi level, and a new transport path opens in the valleys between Coulomb peaks. Until the ground breaking paper of Kuemmeth et al. on spin-orbit coupling in CNTs [11] it was believed that spin and orbital (valley) degrees of freedom could be seen as independent in CNTQDs and the electron spectrum of nanotubes in the absence of magnetic field was considered as fourfold spin-orbital degenerate. A conviction of orbital degeneration was based on the presence of two equivalent dispersion cones $\left(K\right.$ and $\left.K^{\prime}\right)$ in graphene, in nanotubes this degeneracy can be intuitively viewed to originate from two equivalent ways electrons can circle the graphene cylinder, that is clockwise and counter-clockwise [12]. Experimentally the presence of quadruplets of degenerate levels has been confirmed e.g. by observation of fourfold periodicity of the electron addition energy (e.g. [13]). This degeneration also manifests in many-body effects by the enhanced symmetry of many body-resonances. The first 
report on the appearance of the exotic many-body state in CNTs was the result of Jarillo-Herrero et al. indicating the occurrence of Kondo effect of spin-orbital SU(4) symmetry [14]. Later appeared similar reports also evidencing the occurrence of SU(4) Kondo effect in carbon nanotubes [15-19]. Since it is hardly possible from the conductance to distinguish between $\mathrm{SU}(2)$ and $\mathrm{SU}(4)$ symmetries, of importance are ultra-sensitive current noise measurements reported in [18-20]. The problem of simultaneous screening of orbital and spin degrees of freedom has been also discussed from theoretical point of view in several publications [21-43]. Whereas in conventional spin Kondo effect (SU(2)), a formation of many-body dynamical singlet between localized spin and delocalized electrons is a consequence of spin flip cotunneling processes, in the case when spin and orbital degeneracies occur simultaneously $(\mathrm{SU}(4))$, a formation of many-body resonance results from spin, orbital isospin and spin-orbital cotunneling. The spin and orbital degrees of freedom are totally entangled and Kondo resonance for odd occupancies is no longer peaked at the Fermi level $\left(E_{F}\right)$ as in the standard spin Kondo state and Kondo temperature is largely enhanced in comparison to $\mathrm{SU}(2)$ systems. When CNTQD is occupied by two electrons $(N=2)$ there are six degenerate states and Kondo state is formed due to cotunneling induced fluctuations between all these states. Kondo resonance in this case is centered at $E_{F}$. Conclusive observation, confirming that the phenomena reported by Jarillo-Herrero et al. was indeed SU(4) Kondo resonance was detection of splitting of this resonance in parallel magnetic field into four lines. Parallel magnetic field knocks out from degeneracies both spin and orbital isospin and sufficiently strong field destroys Kondo correlations. Some other perturbations suppress the role of only one of the degrees of freedom and then system is left in $\mathrm{SU}(2)$ symmetry and a crossover from Kondo effect of higher symmetry into Kondo effect of lower effect is observed [14,38]. Example of such a case is the action of perpendicular magnetic field, which breaks only spin degeneracy [38]. Similarly valley (orbital) coupling caused by local perturbations introduced e.g. by vacancies or substitution of atoms or by deformations of structure in interatomic distances mix only orbital channels $[44,45]$. At half filling $(N=2)$ parallel magnetic field splits the sixfold degenerate manifold into three doublets and Kondo state of $\mathrm{SU}(2)$ symmetry associated with the lowest doublet emerges $[19,38]$. More recent experiments pointed out on the intrinsic source of breaking of the symmetries and lowering the degeneracy. The use of suspended CNTs [11] allowed to reduce both the disorder in the sample and the dielectric screening due to substrate. The single electron spectroscopy measurements performed on ultraclean nanotubes showed that even at zero magnetic field, the spin and orbital motions are not independent and a level splitting into two Kramers doublets has been observed [11]. This effect has been attributed as resulting from spin-orbit interaction (SO). Destruction of the full spin-orbital entanglement is certainly disadvantageous for quantum computing applications, because storage capacities of qubit systems is smaller than for four-state bit systems associated with $\mathrm{SU}(4)$ symmetry. Also unfavorable effect of SO interaction is opening of a route for spin decoherence. But there is also an advantage of $\mathrm{SO}$ interaction, it gives the way of electrical manipulation of spin degrees of freedom [46,47]. The significant $\mathrm{SO}$ interaction has been confirmed later by other authors [9,40,42-44,48-51]. Almost all of the abovementioned experiments also investigated the Kondo range. The origin of spin-orbit coupling in CNTs is curvature, as was already theoretically predicted by Ando [52] and is described in more detail in [53-55]. In graphene this interaction is almost completely suppressed due to inversion symmetry of graphene plane. In nanotubes inversion symmetry is broken and in consequence the hopping between $p$ orbitals of different parity from different atoms is allowed. The combined effect of curvature and intra-atomic SO coupling mixes spin and orbital channels and the fourfold degenerate manifolds of states are split into doublets by a few tenth of meV. One can look at the source of $\mathrm{SO}$ splitting as the result of effective radial electric field arising out of curvature, which in rotating electron frame is seen as an effective local magnetic field that has opposite direction for the two valleys. Depending on the sign of SO coupling constant it introduces parallel, or antiparallel alignment of spin and angular momentum. The energy of SO coupling is comparable with the energy scale of Kondo effect and therefore the two effects interplay or compete. Several interesting articles have been published taking up this topic $[30,41,51,55-57]$. The first theoretical analysis showing how SO interaction significantly changes the low-energy Kondo physics in CNTQDs was the paper of Fang et al. [56], where the multipeak structure of manybody resonance resulting from the interplay of spin-orbit and Zeeman effect has been analyzed. The most comprehensive study of the impact of SO coupling on Kondo effect is the paper of Galpin et al. [57], where applying numerical renormalization group technique the differential conductance of CNTQD has been calculated as a function of gate voltage and magnetic field. Similar calculations, but additionally taking into account the effect of valley mixing have been presented in $[41,58]$. In [41] also nonlinear transport has been discussed and universality of conductance curves with the energy scale determined by Kondo temperature has been tested. Interesting feature of Kondo correlations reported recently in [41,51] and also explained therein by symmetry arguments, is blocking of those transport resonances that are not related to pseudospin flips.

Our present publication generalizes and supplements the earlier theoretical studies in three aspects:

- we extend the discussion of the interplay of SO interaction and valley mixing in many-body processes by considering additional to disorder, other important sources of valley scattering, not discussed earlier. We analyze the impact of indirect intervalley mixing resulting from the coupling to the leads, where due to interference appears $e^{-h}$ asymmetry within the shell, fact often observed in experiment (e.g. [50]). We also discuss the effect of intervalley mixing induced by short-range Coulomb interactions (valley backscattering - VBS). VBS changes the splitting between different valley and spin states, what can 
be interpreted as a result of the effective spin and intervalley exchange. In effect of this interaction the tendency to form entangled two-electron states competes with Kondo screening of spin or orbital isospin. The reconstruction of dot states resulting from VBS leads in some cases to enhanced degeneracy, what opens the path for the occurrence of high symmetry Kondo effects;

- discussing the effect of magnetic field in CNTQDs, we draw special attention on the role of field induced reconstruction of single electron dispersion curves. This effect is particularly important for narrow gap nanotubes, where the details of the band structure are decisive for the response on the field. This manifests most visibly in the vicinity of the gap. Close to the gap effective spin-orbit coupling changes dramatically and often exhibits different sign for electrons and holes [43]. All these changes strongly modify the conditions for the occurrence of Kondo resonances, what reflects in the gate and field dependencies of conductance and polarization of conductance. We show that for dots in nearly metallic nanotubes the effective spin-orbit splitting can even vanish for some specific values of gate voltages and full SU(4) symmetry could be recovered with all consequences for many-body physics, including possibility of occurrence of SU(4) Kondo effect;

- we generalize the analysis of correlation effects by taking into account the shell mixing processes, that are not negligible for long CNTQDs or high magnetic fields. For specific cases magnetic field partially recovers degeneracy broken by SO interaction, what leads to a formation of the many body resonances of different origins and symmetries. For vanishing intervalley mixing only spin and valley Kondo effects are induced within the single shell. Intershell effects or intervalley mixing additionally allow formation of spin-valley Kondo resonance. Another interesting observation is that for strong Coulomb induced intervalley coupling, or due to intershell coupling, magnetic field can in some cases lead not only two, but also three states to degeneracy. It is interesting from the point of view of quantum computing, because it. would allow to operate in these systems not only on qubits, but also on qutrics (tree state information units). Threefold degeneracy allows also for a formation of Kondo state of exotic SU(3) symmetry, problem previously discussed only in a few works [59-61].

To complement the discussion of the crossovers between Kondo states of different symmetries we present in some cases, apart from evolution of total conductance also the corresponding dependencies of partial conductances, thermopower or noise Fano factor. This is especially useful for analysis of $\mathrm{SU}(4)$ to $\mathrm{SU}(2)$ crossover, because total conductances coincide for both symmetries. Special attention is also paid to spin dependent characteristics important for spintronic applications.

This paper is organized as follows. In Section 2, we describe the model of CNTQD and the many body technique we use in analysis - slave boson approach. Numerical results and analysis are given in Sections 3 and 4, where we successively present the effect of SO interaction on transport characteristics, discuss the impact of different types of intervalley mixing on Kondo physics and analyze the effect of magnetic field and intershell mixing. Section 5 contains summary and a short discussion.

\section{Model and slave boson mean-field formulation}

Our considerations are addressed to the low temperature range and it is enough therefore to restrict to the lowest quadruplet of states labeled by $\operatorname{spin}(\sigma= \pm 1=\uparrow, \downarrow)$ and the orbital pseudospin $\left(m= \pm 1=K, K^{\prime}\right)$ commonly referred to as valley. Although the CNTQD states are in principle additionally numbered by longitudinal momentum $k_{\|}$and circumferential momentum $k_{\perp}$ we do not write them explicitly, because the level separations corresponding to the quantizations of these quantities are much larger than the thermal energy and the energy scale of the processes engaged in many-body effects discussed. Fixed values of $k_{\perp}$ and $k_{\|}$corresponding to the lowest occupied states are assumed, with only one exception in Section 4, where intershell effects are discussed and in this case apart from the lowest also the first excited longitudinal mode is considered. The basic Hamiltonian describing CNTQD is extended two orbital Anderson model:

$$
\mathcal{H}=\mathcal{H}_{L}+\mathcal{H}_{R}+\mathcal{H}_{d}+\mathcal{H}_{t} .
$$

The first two terms describe noninteracting electrons in the leads:

$$
\mathcal{H}_{\alpha}=\sum_{k m \sigma} E_{k \alpha m \sigma} c_{k \alpha m \sigma}^{\dagger} c_{k \alpha m \sigma},
$$

$(\alpha=L, R)$ for the left (right) electrode and $E_{k \alpha m \sigma}$ is the energy of an electron in the lead $\alpha$. The dot Hamiltonian is given by:

$$
\begin{aligned}
\mathcal{H}_{d}= & \sum_{m \sigma} E_{m \sigma} n_{m \sigma}+\mathcal{U} \sum_{m} n_{m \uparrow} n_{m \downarrow} \\
& +\mathcal{U}^{\prime} \sum_{\sigma \sigma^{\prime}} n_{1 \sigma} n_{-1 \sigma^{\prime}}
\end{aligned}
$$

where $n_{m \sigma}=c_{m \sigma}^{\dagger} c_{m \sigma}$ and dot energies are:

$$
\begin{aligned}
E_{m \sigma}^{ \pm}= & \pm \sqrt{\left(-m \frac{E_{g}}{2}+\mu_{o} B_{\|}+\sigma \Delta_{s o}^{1}\right)^{2}+E_{0}^{2}\left(V_{g}\right)} \\
& +m \sigma \Delta_{s o}^{0}+\frac{\sigma \mu_{s} B_{\|}}{2},
\end{aligned}
$$

the upper or the lower sign refer to conduction or valence band states, $E_{g}$ is the bandgap at zero field without spinorbit coupling, $E_{0}$ is gate dependent dot energy for the fixed value of $k_{\|}, B_{\|}$is magnetic field directed along the nanotube axis. SO interaction sets as spin quantization axis the nanotube axis and locks spin and valley degrees of freedom. $\Delta_{s o}^{0}$ and $\Delta_{s o}^{1}$ parameterize the strength of Zeeman and orbital contributions to the spin-orbit coupling [52-54]. The former gives rise to a vertical shift of the Dirac cones that is opposite for two spin directions and 
$\Delta_{\text {so }}^{1}$ gives rise to horizontal shift of Dirac cones, what is equivalent to spin dependent magnetic flux. As it is seen we have included the SO corrections already in the single particle energies (4). They result from SO perturbation of the form [43]:

$$
\mathcal{H}_{s o}=\Delta_{s o}^{1} s_{z} \tau_{\mathbf{x}}+\Delta_{\text {so }}^{0} m s_{z},
$$

where $s_{z}$ is the spin component along the nanotube axis and $\tau_{\mathbf{x}}$ is Pauli matrix in the A-B graphene sublattice space. For large bandgap tubes i.e. in the limit $E_{g} \gg \Delta_{s o}^{0}$, $\Delta_{\text {so }}^{1}$ the SO splitting can be described by one parameter $\Delta=2\left(\Delta_{s o}^{0} \mp \frac{\Delta_{s o}^{1}}{\sqrt{1+\left(E_{0} / E_{g}\right)^{2}}}\right) \approx 2\left(\Delta_{s o}^{0} \mp \Delta_{s o}^{1}\right)$. The terms parameterized by $\mathcal{U}, \mathcal{U}^{\prime}$ describe intra and interorbital Coulomb interactions and the last term $\mathcal{H}_{t}$ describes electron tunneling from the leads to the dot (or vice versa) and takes the form:

$$
\mathcal{H}_{t}=\sum_{k \alpha m \sigma} t\left(c_{k \alpha m \sigma}^{\dagger} c_{m \sigma}+\text { h.c. }\right) \text {. }
$$

Coupling of the dot to the electrodes can be parameterized by $\Gamma_{m \sigma}(E)=2 \pi \sum_{k \alpha}|t|^{2} \delta\left(E-E_{k \alpha m \sigma}\right)$. We assume that $\Gamma_{m \sigma}(E)$ is constant within the energy band, $\Gamma_{m \sigma}(E)=\Gamma$. The full spin-orbital rotational SU(4) symmetry occurs only for $B=0, \Delta=0$ and $\mathcal{U}=\mathcal{U}^{\prime}$. Apart from magnetic field and SO interaction we will also discuss the effect of other symmetry breaking perturbations: direct and indirect valley mixing and valley and spin exchange. The forms of these additional perturbations will be given in the sections, where these problems will be discussed. To analyze correlation effects, we use finite $\mathrm{U}$ slave boson mean field approach (SBMFA) of Kotliar and Ruckenstein [62] and introduce a set of boson operators for each electronic configuration of the single shell of CNTQD. These operators act as projectors onto empty state $e$, single occupied state $p_{m \sigma}$, doubly occupied $d$, triply occupied $t_{m \sigma}$ and fully occupied $f$. The $e$ operators are labeled by orbital index, $p$ operators by indices specifying the corresponding single-electron states, $t$ by indices of state occupied by a hole, and the six $d$ operators denote projectors onto double occupied states $d_{m=1,-1}$ $(\uparrow \downarrow, 0),(0, \uparrow \downarrow)$ and $d_{\sigma \sigma^{\prime}}(\uparrow, \uparrow),(\uparrow, \downarrow),(\downarrow, \uparrow)$ and $(\downarrow, \downarrow)$. To eliminate unphysical states, the completeness relations for the slave boson operators and the conditions for the correspondence between fermions and bosons have to be imposed. These constraints can be enforced by introducing Lagrange multipliers $\lambda, \lambda_{m \sigma}$ and supplementing the effective slave boson Hamiltonian by corresponding terms in (7). The SB Hamiltonian then reads:

$$
\begin{aligned}
\mathcal{H}^{K-R}= & \sum_{m \sigma} E_{m \sigma} n_{m \sigma}^{f}+\mathcal{U} \sum_{m} d_{m}^{\dagger} d_{m}+\mathcal{U}^{\prime} \sum_{\sigma \sigma^{\prime}} d_{\sigma \sigma^{\prime}}^{\dagger} d_{\sigma \sigma^{\prime}} \\
& +\sum_{m \sigma}\left(\mathcal{U}+2 \mathcal{U}^{\prime}\right) t_{m \sigma}^{\dagger} t_{m \sigma}+\left(2 \mathcal{U}+4 \mathcal{U}^{\prime}\right) f^{\dagger} f \\
& +\sum_{m \sigma} \lambda_{m \sigma}\left(n_{m \sigma}^{f}-Q_{m \sigma}\right) \lambda(\mathcal{I}-1) \\
& +t \sum_{k \alpha m \sigma}\left(c_{k \alpha m \sigma}^{\dagger} z_{m \sigma} f_{m \sigma}+\text { h.c. }\right) \\
& +\sum_{k \alpha m \sigma} E_{k \alpha m \sigma} n_{k \alpha m \sigma},
\end{aligned}
$$

with $Q_{m \sigma}=p_{m \sigma}^{\dagger} p_{m \sigma}+d_{m}^{\dagger} d_{m}+d_{\sigma \sigma}^{\dagger} d_{\sigma \sigma}+d_{\sigma \bar{\sigma}}^{\dagger} d_{\sigma \bar{\sigma}}+$ $t_{m \sigma}^{\dagger} t_{m \sigma}+t_{\bar{m} \sigma}^{\dagger} t_{\bar{m} \sigma}+t_{\overline{m \sigma}}^{\dagger} t_{\overline{m \sigma}}+f^{\dagger} f, \quad \mathcal{I}=e^{\dagger} e+$ $\sum_{m \sigma \sigma^{\prime}}\left(p_{m \sigma}^{\dagger} p_{m \sigma}+d_{m}^{\dagger} d_{m}+d_{\sigma \sigma^{\prime}}^{\dagger} d_{\sigma \sigma^{\prime}}+t_{m \sigma}^{\dagger} t_{m \sigma}\right)+f^{\dagger} f$ and $z_{m \sigma}=\left(e^{\dagger} p_{m \sigma}+p_{m \bar{\sigma}}^{\dagger} d_{m}+p_{\overline{m \sigma}}^{\dagger}\left(\delta_{m, 1} d_{\sigma \bar{\sigma}}+\delta_{m,-1} d_{\bar{\sigma} \sigma}\right)+\right.$ $p_{\bar{m} \sigma}^{\dagger} d_{\sigma \sigma}+d_{\bar{m}}^{\dagger} t_{m \sigma}+d_{\bar{\sigma} \sigma}^{\dagger} t_{\overline{m \sigma}}+\left(\delta_{m,-1} d_{\sigma \bar{\sigma}}^{\dagger}+\delta_{m, 1} d_{\bar{\sigma} \sigma}^{\dagger}\right) t_{\bar{m} \sigma}+$ $\left.t_{m \bar{\sigma}}^{\dagger} f\right) / \sqrt{Q_{m \sigma}-Q_{m \sigma}^{2}} \cdot z_{m \sigma}$ renormalize interdot hoppings and dot-lead hybridization (6). The pseudofermion operator $f_{m \sigma}$ is defined by $c_{m \sigma}=f_{m \sigma} z_{m \sigma}$ and the corresponding occupation operator is $n_{m \sigma}^{f}=f_{m \sigma}^{\dagger} f_{m \sigma}$. In the mean field approximation the slave boson operators are replaced by their expectation values. In this way the problem is formally reduced to the effective free-particle model with renormalized hopping integrals and renormalized dot energies. The stable mean field solutions are found from the saddle point of the partition function i.e. from the minimum of the free energy with respect to the mean values of boson operators and Lagrange multipliers. SBMFA best works close to the unitary Kondo limit, but it gives also reliable results of linear conductance for systems with weakly broken symmetry in a relatively wide dot level range, being in a reasonably agreement with experiment and with renormalization group calculations [28,37,63-66]. Current flowing through CNTQD in the $(m \sigma)$ channel can be expressed as $\mathcal{I}_{m \sigma}=\int\left[f_{L}(E)-f_{R}(E)\right] \mathcal{T}_{m \sigma}(E) d E$, where transmission is given by $\mathcal{T}_{m \sigma}=\frac{4 \pi \Gamma_{L} \Gamma_{R}}{\Gamma_{L}+\Gamma_{R}} A_{m \sigma}(E)$ and $f_{\alpha}$ are the Fermi distribution functions. The dot spectral weight $A_{m \sigma}(E)$ is obtained from the retarded Green's function $G_{m \sigma, m \sigma}^{R}$ by $A_{m \sigma}=(-1 / \pi) \operatorname{Im}\left[G_{m \sigma, m \sigma}^{R}\right]$. The linear conductance is defined by $\mathcal{G}_{m \sigma}=\left.\frac{d \mathcal{I}_{m \sigma}}{d V}\right|_{V \rightarrow 0}$ and spin polarization of conductance by $P C_{s}=\left(\sum_{m} \mathcal{G}_{m \uparrow}-\mathcal{G}_{m \downarrow}\right) / \mathcal{G}, \mathcal{G}=\sum_{m \sigma} \mathcal{G}_{m \sigma}$. In an analogous way defined are also polarizations associated with other degree of freedom, orbital $P C_{o}=\left(\sum_{\sigma} \mathcal{G}_{1 \sigma}-\mathcal{G}_{-1 \sigma}\right) / \mathcal{G}$ or spin-orbital Kramers polarizations $P C_{K}=\left(\mathcal{G}_{1 \uparrow}+\mathcal{G}_{-1 \downarrow}-\mathcal{G}_{1 \downarrow}-\mathcal{G}_{-1 \uparrow}\right) / \mathcal{G}$. To get closer insight into evolution of many-body correlations under symmetry breaking perturbations we also analyze other transport quantities. Thermopower (TEP) acts as an excellent tool to describe the crossover between $\mathrm{SU}(4)$ and $\mathrm{SU}(2)$ Kondo

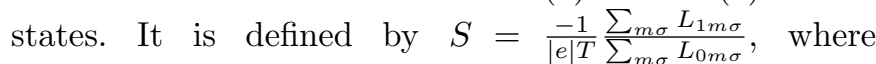
$L_{n m \sigma}=-(1 / h) \int(E-\mu)^{n} \frac{d f}{d E} \mathcal{T}_{m \sigma}(E) d E$. Also the shot noise is strongly affected by a change of correlations and we analyze the noise Fano factor $F$ defined as the ratio between the actual shot noise $\mathcal{S}$ and the Poissonian noise of uncorrelated carriers $(2|e| \mathcal{I})$. The most important information about symmetries of the resonances can be inferred from non-equilibrium noise. This is however outside the formalism we use, but also zero bias noise which can easily be calculated in SBMFA formalism distinguishes between different symmetry classes. In the linear regime noise can be expressed by transmissions alone nd the linear Fano factor then reads $F=\sum_{m \sigma} \mathcal{T}_{m \sigma}\left(1-\mathcal{T}_{m \sigma}\right) /\left(\sum_{m \sigma} \mathcal{T}_{m \sigma}\right)$. We parameterize the unperturbed fully spin-orbital rotationally invariant CNTQD Hamiltonian by three parameters: charging energy $\mathcal{U}$, tunnel rate between the dot and the reservoirs $\Gamma$, and the half-bandwidth $D$. The numerical results discussed below are presented with the 

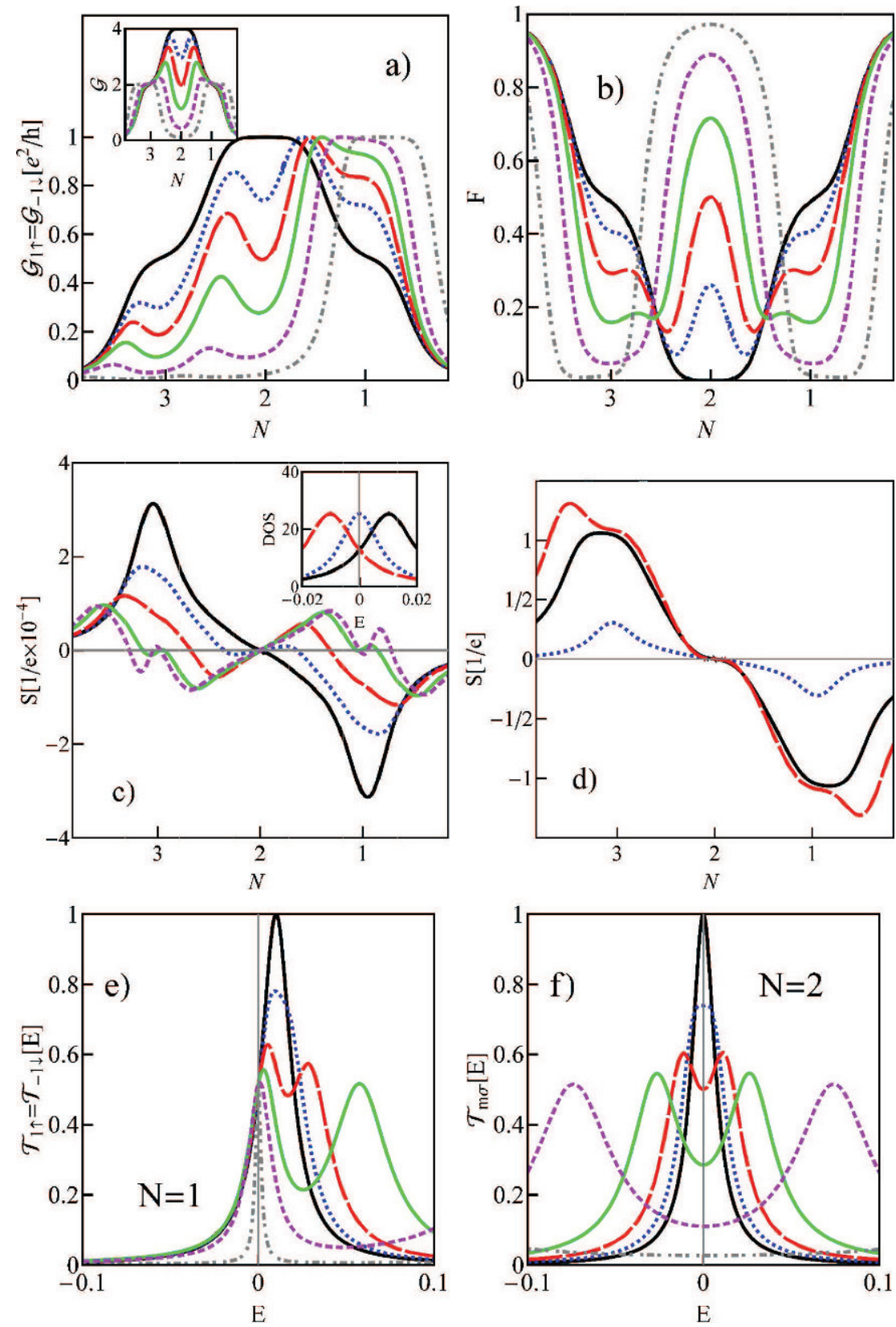

Fig. 1. Transport characteristics of CNTQD with SO interaction. (a) Spin and orbital resolved conductances of CNTQD vs dot occupation $N=(1 / 2)\left(1-2 E_{0} / \mathcal{U}\right)$ plotted for several values of SO splitting $\Delta: \Delta=0$ (solid black line), $\Delta=0.025$ (dotted blue), $\Delta=0.05$ (dashed red), $\Delta=0.1$ (solid green), $\Delta=0.25$ (short dashed purple) and $\Delta=0.75$ (dashed dotted grey). The same assignment of the lines is valid also for the inset and figures (b,e,f). Inset presents corresponding total conductances. Parameters: $\mathcal{U}=3$ and $\Gamma=0.05$ (if not specified differently the same parameters apply also to other pictures). (b) Noise Fano factors. (c) Thermopower for $\Delta=0$ (solid black), $\Delta=0.08$ (dotted blue), $\Delta=0.2$ (dashed red), $\Delta=0.5$ (solid green), $\Delta=0.65$ (short dashed purple) $\left(V=0.005\right.$ and $\left.T=10^{-6}\right)$. (d) Temperature evolution of thermopower for $\Delta=0$ and $T=2 \times 10^{-2}$ (dashed line), $T=10^{-2} \approx T_{K}$ (solid black), $T=10^{-3}$ (dotted blue) $(V=0.005)$. (e, f) Partial transmissions for $N=1$ and $N=2$ plotted for different values of SO splitting.

use of energy unit defined by its relation to the bandwidth $2 D=100$. The assumed energy unit corresponds to energy of $1-4 \mathrm{meV}$. If not specified differently the results are presented for $\mathcal{U}=3$ and $\Gamma=0.05$. Typically charging energy for semiconducting CNTs is of order of tens of $\mathrm{meV}[67,68]$ and $\Gamma$ in the assumed weak-coupling regime is of order of several meV $[14,15,69]$.

\section{Single-shell many-body effects}

\subsection{Breaking of SU(4) symmetry by spin-orbit interaction}

The fully symmetric system characterized by SU(4) unitary group of spin-orbital rotations $\left(\mathcal{U}=\mathcal{U}^{\prime}, \Delta=0\right.$ and 

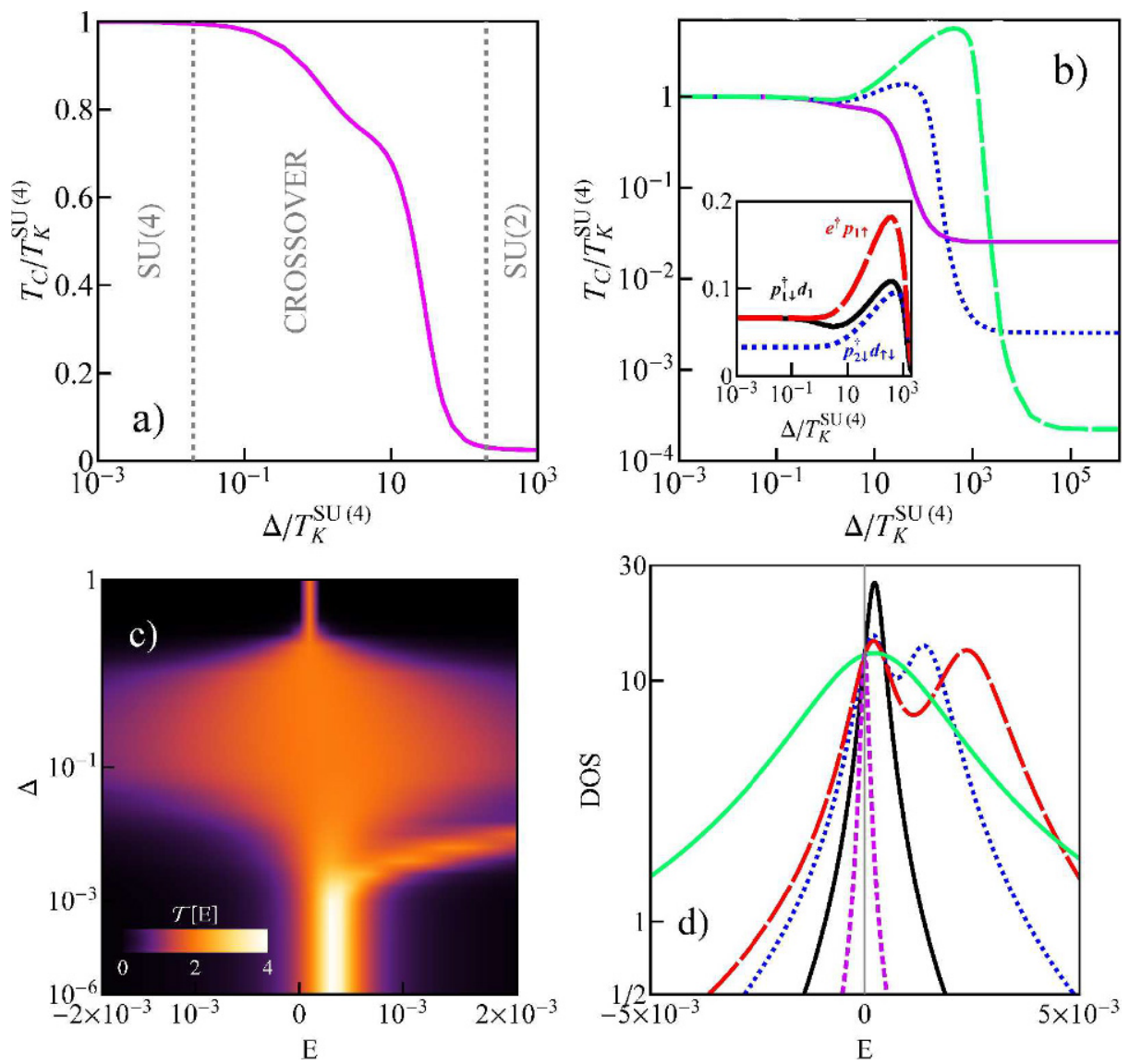

Fig. 2. Spin-orbit interaction induced $\mathrm{SU}(4) \rightarrow \mathrm{SU}(2)$ crossover in the single electron valley $(N=1)$. (a) Characteristic temperatures $T_{C}$ of many-body resonances vs SO splitting $\Delta(U=3)$. (b) $T_{C}(\Delta)$ for $\mathcal{U}=3$ (solid line), $\mathcal{U}=4$ (dotted), $\mathcal{U}=5$ (dashed). Inset shows mean values of the product of slave boson operators plotted vs SO splitting parameter. (c) Transmission map $\mathcal{T}(E, \Delta)$ for $\mathcal{U}=5$. (d) Densities of states of many-body resonances for $\Delta=0$ (solid black line), $\Delta=0.003$ (dotted blue), $\Delta=0.005$ (dashed red), $\Delta=0.1$ (solid green) and $\Delta=0.52$ (short dashed purple).

$B=0)$ exhibits the fourfold degeneracy for odd occupancies $(N=1,3)$ and sixfold degeneracy at half filling $(N=2)$. CNT Hamiltonian is in this case invariant under time-reversal and valley-reversal. For $N=1$ (1e valley) the degenerate states are $\left(|m \sigma\rangle, m=K, K^{\prime}, \sigma=\uparrow, \downarrow\right)$ and for $N=3\left(|\underline{m \sigma}\rangle, \underline{m}=K, K^{\prime}, \underline{\sigma}=\uparrow, \downarrow\right)$, where the underlined quantum numbers refer to the unoccupied states i.e. $|K \uparrow\rangle$ for instance denotes the state $\left|K \downarrow K^{\prime} \uparrow K^{\prime} \downarrow\right\rangle$. In the following we will also interchangeably use instead of $K, K^{\prime}$ quantum numbers $1,-1$. The six degenerate twoelectron states for $N=2$ are $\left|K \uparrow K^{\prime} \downarrow\right\rangle,|K \uparrow K \downarrow\rangle, \mid K^{\prime} \uparrow$ $\left.K^{\prime} \downarrow\right\rangle,\left|K \uparrow K^{\prime} \uparrow\right\rangle,\left|K \downarrow K^{\prime} \downarrow\right\rangle,\left|K \downarrow K^{\prime} \uparrow\right\rangle$. For the Kondo effect to occur apart from degeneracy it is also required that spin and valley quantum number are conserved. It may be approximately satisfied if the contacts constitute parts of the same CNT as the dot and the carriers dwell for some time before moving into the metallic electrodes. One can think then that before and after tunneling process electron share the same degrees of freedom [24,41]. Kondo effect in odd electron valleys results from effective spin and valley isospin fluctuations (transitions between four degenerate states) occurring due to cotunneling processes. Both the total spin $S_{Z}=\left(n_{\uparrow}-n_{\downarrow}\right) / 2$ and orbital pseudospin $T_{Z}=\left(n_{1}-n_{-1}\right) / 2$ are quenched by these fluctuations. The spin-valley many-body peak is shifted above $(1 e)$ or below $(3 e)$ the Fermi level corresponding to phase shifts $\delta=\pi / 4$ and $\delta=3 \pi / 4$ respectively. Increased degeneracy reflects in broadening of Kondo resonance in comparison to the standard spin Kondo peak, what means exponential enhancement of Kondo temperature. In $2 e$ valley for $\Delta=0$ transitions between all six states lead to a formation of Kondo resonance centered at $E_{F}$, the corresponding phase shift is $\delta=\pi / 2$ and conductance is doubled in comparison to SU(4) Kondo effect for odd number of electrons. Spin-orbit coupling depends on chirality and diameter. The observed values of SO splitting are of order of $0.1-0.4 \mathrm{meV}[11,43,50]$, but also splitting of up to $3.4 \mathrm{meV}$ has been reported [9]. Figure 1 presents linear partial conductances, noise Fano factor, transmissions and thermoelectric power plotted for different values of SO coupling. In all these quantities the crossover between different symmetries induced by SO interaction is evidently reflected. Total conductance curves are given only as a point of reference in the inset of Figure 1, but we skip an analysis of their evolution with the increase of SO coupling, because this problem has been discussed 


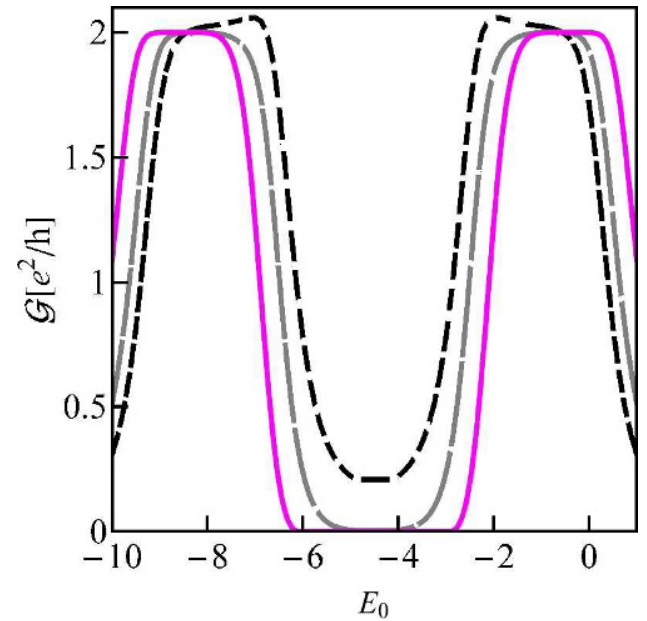

Fig. 3. Direct valley mixing. Conductances of CNTQD vs unperturbed dot energy level $E_{0}$ for $\Delta=0.5$ and valley mixing parameters: $\Delta_{K K^{\prime}}=0$ (short dashed line), $\Delta_{K K^{\prime}}=0.3$ (dashed) and $\Delta_{K K^{\prime}}=0.8$ (solid).

earlier in $[30,58]$. It is also known that the total conductance is not a proper quantity to track the crossover between the considered symmetries, because both symmetries $\mathrm{SU}(4)$ and $\mathrm{SU}(2)$ achieve the same unitary limit in the Kondo range for odd electron occupations. For $N=2$ conductance is doubled in comparison to the odd case, what is a consequence of half transmission of each channel in odd valleys and full transmission for even occupation (Figs. 1c and 1d). In the latter case tunnel induced fluctuations between six two-electron states lead to a formation of Kondo resonance centered at the Fermi level. Different opening of the many-body transmission channels is clearly seen in different limits of spin and orbital resolved conductances in odd and even valleys (Fig. 1a). This is also reflected in the linear noise. Perfect transmission of all channels in $2 e$ valley corresponds to no noise $(F=0$, Fig. 1b). In odd valleys on the other hand, the observed half transmission of spin-orbital channels corresponds to strong partition, what means strong shot noise $(F=1 / 2)$ $[18,20,37]$. Total conductance curve and noise Fano factor are symmetric vs $E_{0}=-(3 / 2) \mathcal{U}$ line (symmetry of 1 e and 3e regions), as they reflect transmission values at $E_{F}$ for $T=0$ or transmissions averaged over the thermal energy $k_{B} T$ for higher temperatures. Thermopower complements this information. This quantity provides information on the possible asymmetry of the transmission line $\mathcal{T}(E)$ near the Fermi energy in the range of the thermal broadening. In the limit of $T \rightarrow 0$ the sign of TEP reflects the slope of the spectral function at the Fermi level. For $N=1$ Kondo resonance lies above $E_{F}$ (inset of Fig. 1c), the slope is positive, resulting in a negative TEP. For $N=3$ the peak is located below $E_{F}$ and the opposite sign is observed. Zero value of thermopower for $N=2$ corresponds to the central position at the Fermi energy of Kondo resonance for even occupation. Now let us look at the broken symmetry case induced by spin-orbit interaction $(\Delta \neq 0)$. For $N=2$ the sextet is split into three groups of states, of degeneracy 1, 4 and 1, with relative energies $-\Delta, 0$, and $\Delta$ respectively (Fig. 5f). Since the state of the lowest energy is singlet, Kondo correlations are gradually destroyed by SO interaction, what results in a drop of conductance. In transmission, the top of the peak is replaced by a valley, deepening with the increase of $\Delta$ and since this effect occurs around the Fermi level and it manifests by a change of the sign of TEP in the $N=2$ region. Spin-orbit interaction lowers the symmetry, but it does not break time-inversion symmetry. Consequently in odd valleys it splits the four degenerate levels into the pairs of Kramers doublets separated by an energy $\Delta$ (Fig. 5e) and the system gradually falls into $\mathrm{SU}(2)$ symmetry class with the increase of SO coupling. Only lower doublet in the single electron range $|K \downarrow\rangle,\left|K^{\prime} \uparrow\right\rangle$ or the higher doublet $|K \uparrow\rangle,\left|K^{\prime} \downarrow\right\rangle$ for triple occupancy remains active in Kondo fluctuations. Evolution of partial conductances is illustrated in Figure 1a, taking as the examples spin and orbital resolved conductances for the channels corresponding to the lower Kramers doublet. In the $\mathrm{SU}(4)$ limit $(\Delta=0) \mathcal{G}_{1-}=\mathcal{G}_{-1+}=1 / 2$ for $N=1$ corresponding to a quarter occupancy of each spin-orbital. Increase of $\Delta$ results in the increase of the partial conductances, for $\Delta \gg T$ they reach the unitary $\mathrm{SU}(2)$ Kondo limit. The curves $\mathcal{G}_{1+}=\mathcal{G}_{-1-}$, not presented here (higher Kramers doublet), are symmetric under shell electron-hole symmetry line and these transport channels are almost completely closed in $N=1$ range for strong SO coupling, they are open however in $3 e$ valley. For this filling, in turn, as seen in Figure $1 \mathrm{a}, \mathcal{G}_{1-}$ and $\mathcal{G}_{-1+}$ are negligible. Reduction of symmetry with the increase of SO coupling manifests itself in a tendency to total suppression of TEP at the points $N=1,3$, which indicates the location of the $\mathrm{SU}(2)$ Kondo resonances at the Fermi energies. Local minima of maxima of thermopower curves visible for small deviations from these integer occupations reflect the influence of many-body fluctuations in Kondo active doublets. Similar local extremes are also seen closer to the Coulomb border lines and in this case they signal the impact of charge fluctuations. Figure 1d illustrates temperature evolution of thermoelectric power shown only for the full SU(4) symmetric case. Apart from an obvious increase of TEP, a shift of extremes to the borders of Coulomb blockade regions is observed for temperatures above $T_{K}$, i.e. in the range, where Kondo correlations are destroyed. Destruction of the symmetry or the crossover between the symmetry classes can be also monitored by shot noise (Fig. 1b). Killing of Kondo correlations at half filling for strong enough SO coupling causes that the noise gets Poissonian. For odd occupations the $\mathrm{SU}(4)$ symmetry transmission is distributed evenly across all channels and they are only partially open. When system evolves into $\mathrm{SU}(2)$ class two of the channels are closed and two other become transparent. In consequence Fano factors change from $F=1 / 2$ for $\mathrm{SU}(4)$ symmetry to $F=0$ for $\mathrm{SU}(2)$. In the latter case electrons pass through the dot with probability one, and the linear conductance is noiseless. We are aware that much more information for understanding the $S U(4) \rightarrow S U(2)$ crossover would provide analysis of nonlinear noise, but that would require going beyond SBMFA formalism. Discussion of nonlinear noise for CNTQDs have been done for Kondo-correlated 

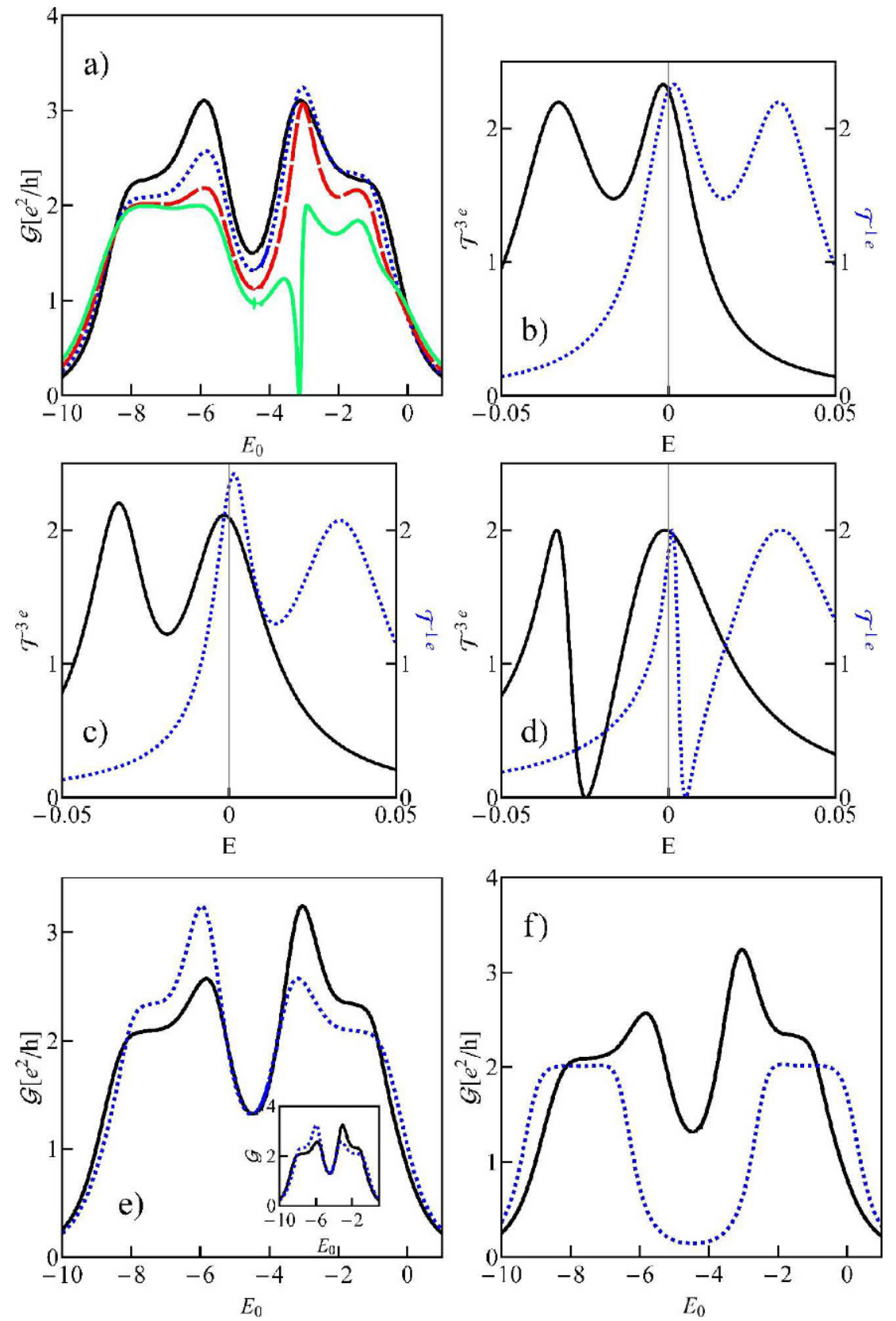

Fig. 4. Indirect valley mixing. (a) Conductance of CNTQD disturbed by direct and indirect valley mixing, $\Delta=0.1, \Delta_{K K^{\prime}}=0.01$ and $q=0$ (solid black line), $q=1 / 2$ (dotted blue), $q=3 / 4$ (dashed red), $q=1$ (solid green). (b,c,d) Transmissions for $N=1$ (dotted line) and $N=3$ (solid line) $\Delta=0.1, \Delta_{K K^{\prime}}=0.01$ and $q=0$ (b), $q=1 / 2$ (c), $q=1$ (d). (e) Intershell $e-h$ asymmetry: conductance for $\Delta=0.1$ and $\Delta_{K K^{\prime}}=0.01, q=-1 / 2$ (dotted blue line), $\Delta_{K K^{\prime}}=0.01, q=1 / 2$ (solid). Inset presents conductances for $\Delta_{K K^{\prime}}=-0.01, q=1 / 2$ (dotted blue line), $\Delta_{K K^{\prime}}=0.01, q=1 / 2$ (solid). (f) Comparison of conductances for $\Delta<T_{K}(\Delta=0.1$, solid line $)$ with the case $\Delta \gg T_{K}(\Delta=1$, dotted line $), \Delta_{K K^{\prime}}=0.01, q=1 / 2$.

CNTQD [18-20] but only for the case undisturbed by SO interaction. It was shown how fluctuations affect the residual interactions between the quasiparticles, what leads to a clear shot noise enhancement for SU(4) systems and it distinguishes it from $\mathrm{SU}(2)$ case, for which shot noise reduction is expected $[18,19,70,71]$. Figure 2a shows characteristic temperature $T_{C}$ of many-body resonances in $N=1$ region, vs SO splitting $\Delta$ and Figure $2 \mathrm{~b}$ presents $T_{C}(\Delta)$ for several values of Coulomb interaction.
$T_{C}$ has been estimated from the temperature dependence of conductance $\left(\mathcal{G}\left(T_{C}\right)=\mathcal{G}(0) / 2\right)$ or from the position of the center of the peak $(\widetilde{\epsilon})$ and half width of the resonance peak $(\widetilde{\Delta}), T_{C}=\sqrt{\widetilde{\epsilon}^{2}+\widetilde{\Delta}^{2}}$, and both estimations are qualitatively consistent and show the same tendency. The stronger enhancement of SU(4) Kondo temperature with respect to $\mathrm{SU}(2)$ Kondo temperature is observed for larger values of $\mathcal{U}$, what is consistent with the well known exponential dependencies of $T_{K}$ on $\mathcal{U}$ combined with the 

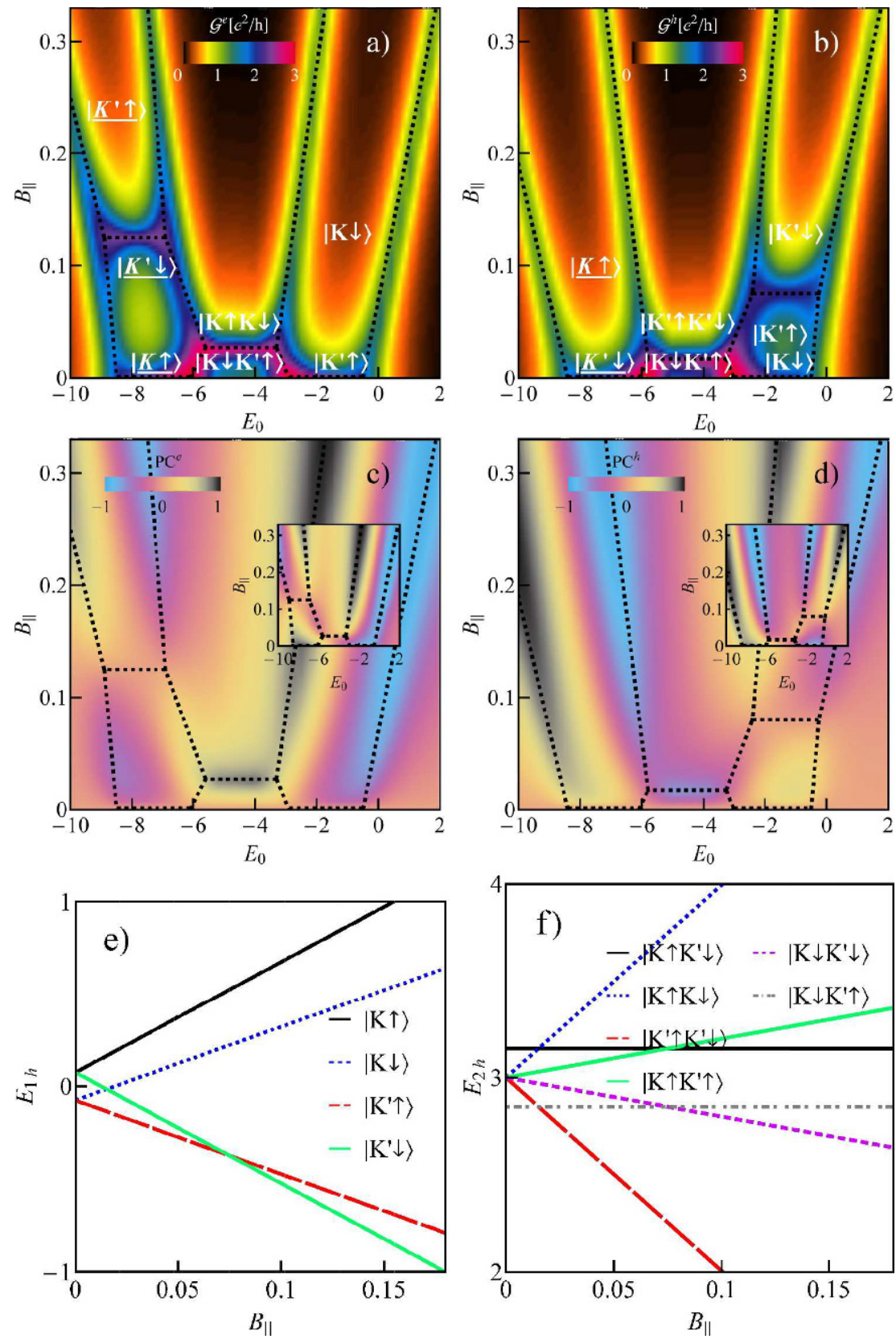

Fig. 5. CNTQD in parallel magnetic field $\left(\Delta_{s o}^{1}=-0.01, \Delta_{s o}^{0}=0.04, \mu_{o} / \mu_{s}=5\right)$. (a) Electron conductance map $\mathcal{G}^{e}\left(E_{0}, B_{\|}\right)$ with the ground states stability regions on it. (b) The same as in (a) but for holes. (c) Spin polarization of electron conductance and valley conductance polarization in the inset. (d) The same as in (c), but for holes. (e) Field dependence of single hole states. (f) Field dependence of two-hole states.

dependence on the fold of degeneracy [72]. The crossover region also extends with increasing $\mathcal{U}$ and interestingly, the characteristic temperatures do not always evolve monotonically. For high $\mathcal{U}$ values, where pure $\mathrm{SU}(4)$ resonances are relatively narrow, the effect of symmetry breaking induced broadening is more significant than for wider SU(4) Kondo resonances and this effect dominates in a broad range of crossover region over the tendency for extracting the additional peak associated with the processes encountered in the doublet inactive in $\mathrm{SU}(2)$ Kondo fluctuations (Fig. 2d). The region of enhanced characteristic temperature corresponds to mixed valence range, where charge fluctuations become also of importance. In SBMFA formalism it manifests for discussed here $1 e$ valley in enhancement of mean values of $p$ and $e \mathrm{SB}$ operators in the range of enhanced $T_{C}$. The products of operators presented in the inset of Figure $2 \mathrm{~b}$ are the dominant contributions to SB parameters $z_{m \sigma}$, which determine the widths of the resonance. The enormous broadening of many-body resonance in the crossover region is also visualized on the $\mathcal{T}(\Delta, E)$ transmission map (Fig. 2c). 


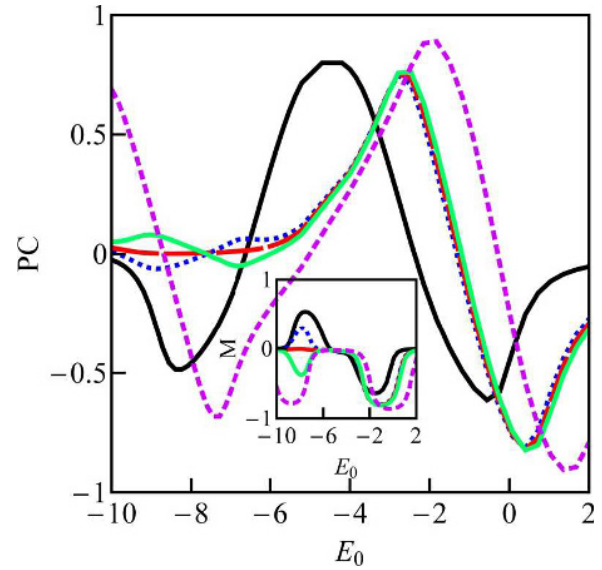

Fig. 6. Spin polarization of conductance for the chosen values of magnetic field: $B_{\|}=0.025\left(B_{o}\right)$ (solid black line), $B_{\|}=$ 0.118 (dotted blue), $B_{\|}=0.125\left(B_{s}\right)$ (dashed red), $B_{\|}=0.13$ (solid green), $B_{\|}=0.278$ (short dashed purple). Inset presents magnetic moments at the dot for the same choice of the fields.

\subsection{Direct and indirect valley mixing}

Apart from lifting of the fourfold degeneracy by SO interaction, some experiments indicate that a similar role can be played by valley mixing [11,41]. To scatter from one valley to the other, a scattering vector of the order of the Brillouin zone vector is needed. This can be caused by scattering on nonmagnetic impurities, structure defects or deformations of structure e.g. by putting CNT on a rough substrate. Valley mixing can be modeled by $\sum_{m} \Delta_{m,-m} c_{m \sigma}^{\dagger} c_{-m \sigma}$. Spin-orbit interaction reduces degeneration, but does not change the quantum numbers, valley mixing preserves only spin. Due to the time inversion symmetry double degeneracy is preserved in odd valleys. Splitting between the doublets caused by $\mathrm{SO}$ interaction and valley mixing is given by $\Delta_{e f f}=\sqrt{\Delta^{2}+\Delta_{K K^{\prime}}^{2}}$. Figure 3 shows the gate voltage dependence of conductance for fixed value of SO coupling presented for several values of $\Delta_{K K^{\prime}}$. Valley mixing enhances the SO destruction of Kondo correlations at half filling. The borders of odd occupation valleys and Kondo conductance plateaus move symmetrically away from shell $e-h$ symmetry point with the increase of $\Delta_{K K^{\prime}}$. Since valley scattering has been detected also in ultraclean nanotubes $[11,41,43,45]$, it is worth considering also the role of other mechanisms that cause valley mixing. One of them is local Coulomb scattering, which we will discuss in Section 3.4. Also electrical contacts can induce valley scattering due to valley mixing during tunneling. Commonly accepted conviction on valley conservation during tunneling, which is also adopted in this work, is based on the view, that the leads to the dot are formed within the same nanotube as the dot, or that electrons from metallic leads enter the nanotube segment before tunneling. If, however, these requirements are not fully met, some mixing in the orbital channels may occur. The effect of contacts which may mix orbital numbers is thoroughly discussed in $[41,43]$. Indirect valley scattering via the electron states of electrodes can be represented by the off diagonal terms of electrode-dot coupling matrix $\Gamma_{m,-m}$, and following $[73,74]$ they can be approximated by $\Gamma_{m,-m}=i q \Gamma$. The processes represented by off diagonal terms of $\Gamma$ result from various interference effects and are the consequence of indirect transitions between dot orbitals by states in the electrodes. Parameter $q$ describes the strength of this indirect mixing, in general $q$ can be a complex number, but in our considerations is assumed real $|q|<1$. In the case discussed at present, besides the cotunneling processes, which preserve valley also cotunneling, which flips isospin comes into play in formation of many-body resonances. The partially separated peaks of transmissions presented in Figures 4b-4d correspond to resonances set up primarily by cotunneling processes within only one of the Kramers doublets for each of the resonances, although for small SO splitting also the states from the second doublet play some role. The peaks located around $E_{F}$ correspond to Kondo like resonances. In $1 e$ valley the Kondo peak is lower in energy than the peak associated with tunneling into the second doublet and in $3 e$ valley the opposite energy ordering of the peaks occurs. Due to the electrode mediated destructive interference between the Kondo state and the renormalized states from the second doublet the asymmetric Fano resonance is formed between the peaks. As it is seen Fano resonance differently perturbs Kondo peak in $1 e$ and $3 e$ valleys and this results in intrashell $e^{-h}$ asymmetry $(1 e-3 e)$ of conductance (Fig. 4a). Formally changing the sign of one of the parameters either $\Delta_{K K^{\prime}}$ or $q$ on the opposite results in reversing of the asymmetry, conductances of $1 e$ and $3 e$ valleys change the role. Similar interference induced asymmetry effects have been earlier reported e.g. for double dot Kondo systems [74]. In carbon nanotubes the single shell $e^{-} h$ conductance asymmetry has been observed in many systems $[14,41,75]$. Of course experimentally observed conductance asymmetry between $1 e$ and $3 e$ valleys can be also caused by many other reasons, which we do not discuss here, e.g. asymmetry in coupling of the leads to different SO Kramers doublets. Also the gate dependence of dot-lead coupling, or gate dependence of spin-orbit interaction can introduce intrashell $1 e-3 e$ asymmetry, but for the narrow energy interval of the single shell, the latter effects are expected to be of minor importance at least for wide gap tubes. As we discuss in Section 3.5 they might be of importance in nearly metallic nanotubes. In the limit of strong indirect intervalley mixing $(q=1)$ the destructive interference leads to a zero transmission dip (Fig. 4d). For certain values of gate voltage this dip locates at the Fermi energy and linear conductance vanishes in this case. The largest asymmetry caused by indirect valley mixing is observed in the crossover region between $\mathrm{SU}(4)$ and $\mathrm{SU}(2)$ symmetries, for $\Delta \gg T_{K}^{S U(4)}$ conductance again becomes approximately symmetric with respect to $E_{0}=-(3 / 2) \mathcal{U}$ line (Fig. 4f).

\subsection{Magnetic field}

Analysis of transport through strongly correlated CNTQD in magnetic field, which takes into account SO coupling has been already discussed in literature $[9,30,41,43,58]$. Here we only supplement the earlier reports by presenting 

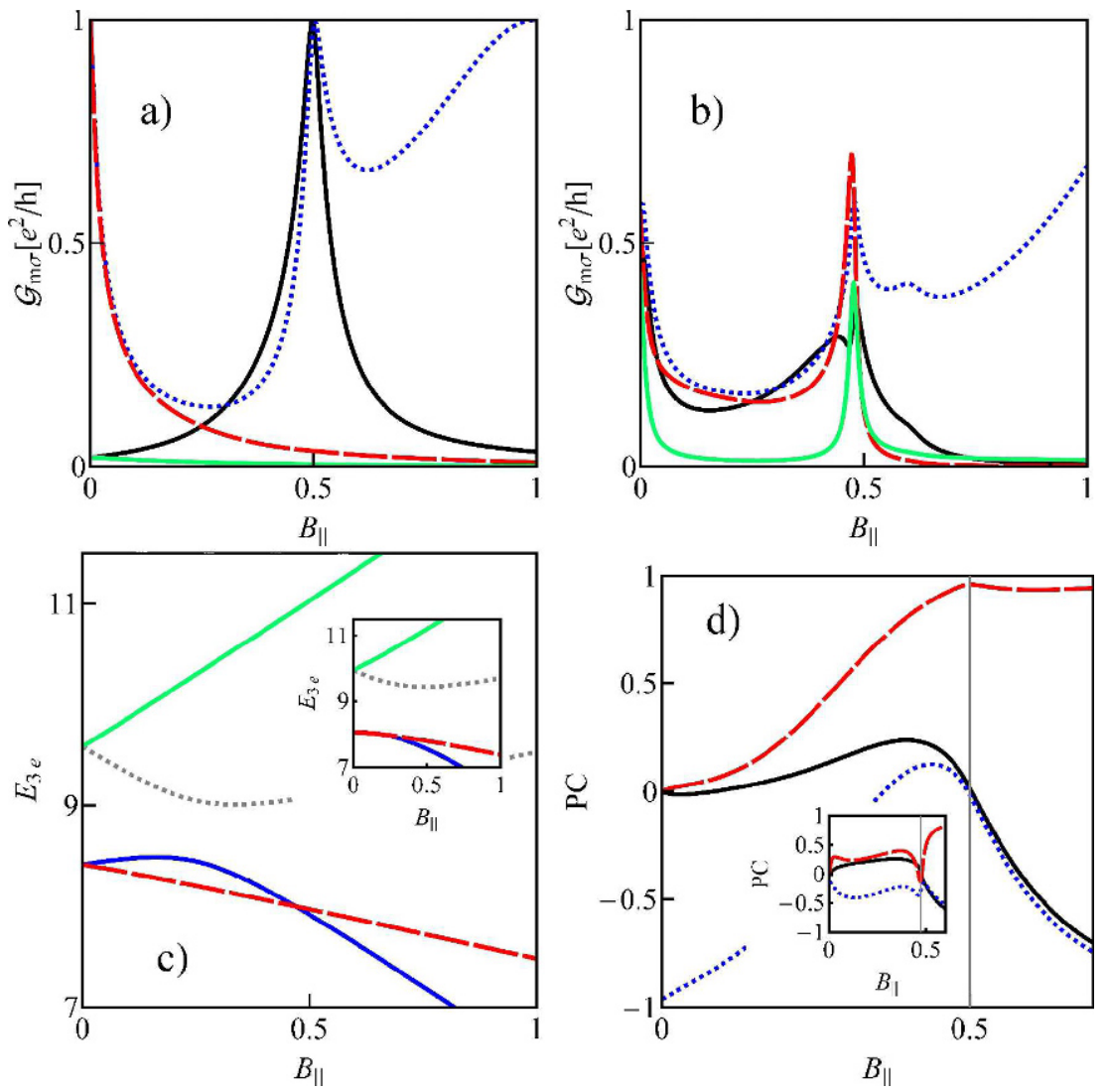

Fig. 7. Impact of valley scattering on field dependencies. (a) Field dependencies of spin-orbital resolved conductances of CNTQD for $N=3$. (a) $\Delta_{K K^{\prime}}=0, \mathcal{G}_{1 \uparrow}$ (solid black line), $\mathcal{G}_{1 \downarrow}$ (dotted blue), $\mathcal{G}_{-1 \uparrow}$ (dashed red), $\mathcal{G}_{-1 \downarrow}$ (solid green). (b) The same as in (a), but for $\Delta_{K K^{\prime}}=0.3$. (c) Field dependencies of three-electron states for $\Delta_{K K^{\prime}}=0.3$ and for $\Delta_{K K^{\prime}}=0.8$ in the inset. (d) Polarizations of conductance for $N=3$ : spin conductance polarization (solid line), valley polarization (broken), Kramers polarization (dotted) for $\Delta_{K K^{\prime}}=0$ and for $\Delta_{K K^{\prime}}=0.3$ in the inset.

conductance map also for holes and discussing polarization. In order to facilitate the latter discussion we complement the conductance maps by the ground state configuration diagram of the system (Figs. 5a and 5b). Magnetic field breaks time-inversion symmetry, parallel magnetic field splits the Kramers doublets in odd valleys and splits the quartet in $2 e$ valley by both spin and orbital Zeeman effects (Figs. 5e and 5f). For magnetic field of energy exceeding Kondo energy the corresponding manybody correlations are destroyed and as a consequence the linear conductance is suppressed. Let us focus on the hole case, for electrons the picture is analogous, replaced is only the role of single occupied and triple occupied valleys. Figure 5e shows single-hole energies. Hereafter presenting electron or hole energies we use the shifted energies defined by $E_{1 e(1 h)}(m \sigma)=E_{m \sigma}^{ \pm}-E_{0}^{ \pm}$, where $E_{0}^{ \pm}= \pm \sqrt{E_{g}^{2}+E_{0}^{2}}$. As it is seen in Figure 5e a competition of SO interaction and Zeeman effect in $1 h$ valley leads to level crossing in the ground state. For the field $B_{s}=\Delta^{h} / g \mu_{s}(N=1)$ (Fig. 5e), the energy of the state $\left|K^{\prime} \uparrow\right\rangle$ is crossed by energy line of one of the states from excited Kramers doublet $\left|K^{\prime} \downarrow\right\rangle$ i.e. degeneracy is recovered. This allows revival of the Kondo effect. Cotunneling induced spin fluctuations are responsible for the creation of the many-body resonance in this case (spin $\mathrm{SU}(2)$ Kondo effect). Similar field induced recovery of degeneracy is observed in the $N=2$ sector (Fig. 5f), where at the field $B_{o}=\Delta^{h} / g \mu_{o}$ ground state $\left|K \uparrow K^{\prime} \downarrow\right\rangle$ crosses $\left|K^{\prime} \uparrow K^{\prime} \downarrow\right\rangle$ state. The corresponding quantum fluctuations leading to Kondo effect are now valley isospin fluctuations and spin is preserved (valley $\mathrm{SU}(2)$ Kondo effect). In the wide gap limit here discussed, $E_{G}^{0} \gg \Delta_{s o}^{0}, \Delta_{s o}^{1}$ and for $\Delta_{K K^{\prime}}=0$ the Kondo lines of enhanced conductance are parallel to the gate voltage axis, because the corresponding characteristic fields $B_{o}$ and $B_{s}$ are determined by SO splitting alone. Comparing conductance maps for electrons and holes (Figs. 5a and $5 \mathrm{~b}$ ) it is seen that electron-hole symmetry around the band gap is broken. The reason is that orbital-like contribution to SO coupling $\Delta_{s o}^{1}$ contributes with opposite signs for electrons and holes. Looking at the areas of stability of states presented in Figures 5a and 5b one can see that neighboring Coulomb blockade regions differ in magnetic moments and this reveals by an increase of polarization of conductance at the borders of Coulomb blockade regions (Figs. 5c and 5d). The corresponding lines for electrons and holes are characterized by polarizations of opposite signs. Conductance on the Kondo lines is unpolarized in odd valleys and polarized in even. In odd valleys it is the spin, which due to cotunneling processes, despite applied 

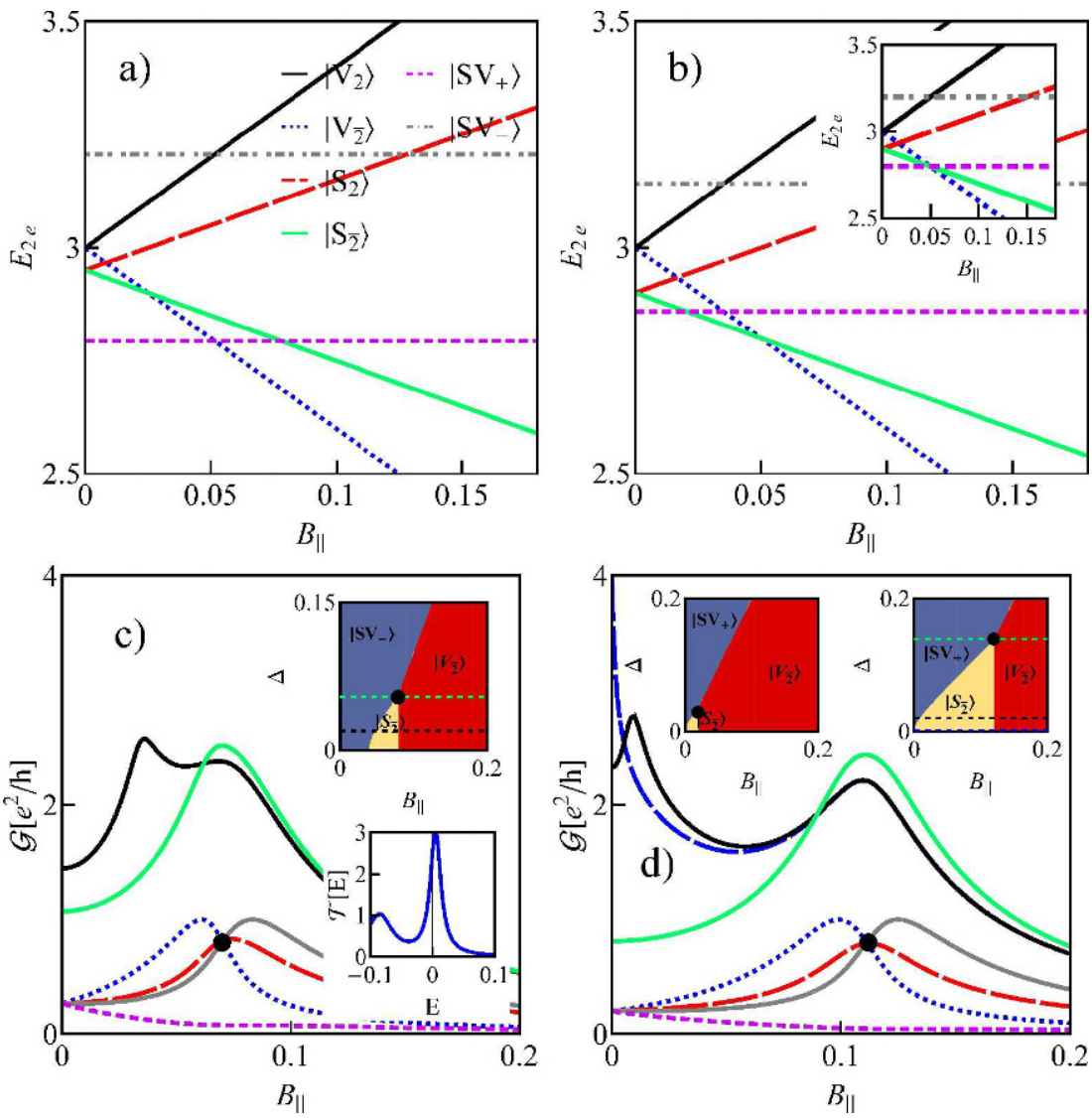

Fig. 8. Coulomb Intervalley scattering. (a) Field dependencies of two-electron states of CNTQD in the case of weak intervalley scattering $(V=0.05, \Delta=0.1)$ and (b) for strong intervalley scattering $(V=0.1, \Delta=0.05)\left(\mathcal{U}=3\right.$ and $\left.\mathcal{U}^{\prime}=2.8\right)$. Inset of Figure $8 \mathrm{~b}$ illustrates the field induced threefold degeneracy of two-electron states occurring for $V=0.1$ and $\Delta=0.087$. (c) Total conductance for $V=-0.04$ and $\Delta=0.02$ (solid black) and for $\Delta=0.053$ (solid green) with the spin-orbital resolved contributions (solid gray line $(-1 \uparrow)$, dashed red $(1 \downarrow)$, dotted blue $(1 \uparrow)$ and dashed magenta $(-1 \downarrow)$ ). Upper inset shows the ground-state diagram. Dashed lines on the inset mark $\Delta$ values, for which the conductances are plotted and black point marks the threefold degeneracy. Lower inset on Figure 8c presents transmission in the SU(3) Kondo state. (d) Conductance for $V=0.04 \Delta=0$ (dashed blue line) and $\Delta=0.02$ (solid black) and for $\Delta=0.127$ (solid green). Insets show the ground-state diagrams for (left: $\mathcal{U}=\mathcal{U}^{\prime}=3$ ) and (right: $\mathcal{U}=3$ and $\mathcal{U}^{\prime}=2.8$ ). Lower curves present the spin-orbital resolved conductances for the $\mathrm{SU}(3)$ symmetry.

magnetic field, freely fluctuates between two orientations and orbital pseudospin is fixed. Transport occurs effectively only in one orbital channel with equal probability for both spin orientations and thus conductance is spin unpolarized and orbitally (valley) polarized in this case (compare a map of valley polarization presented in the insets of Figures 5c and 5d). For half filling on the other hand, orbital pseudospin fluctuates and spin orientation is fixed, what results in spin polarization of conductance. The orientations of net spin magnetic moments of Kondo active doublets are opposite for $2 e$ and $2 h$ occupations and correspondingly opposite are also spin polarizations of conductance in these regions. Conductance is orbitally unpolarized in this case. Figure 6 presenting spin polarization vs gate voltage for several values of magnetic field highlights the possible spintronic applications. Polarization of conductance can be changed both by magnetic field and gate voltage. The former is a consequence of the field dependence of gate voltages at which the Coulomb borders occur and electrical control is due to subsequent crossing of different Coulomb borders with the change of the gate. Finally let us look at the impact of valley scattering on transport characteristics in magnetic field. When both SO coupling and valley mixing are taken into account the field dependencies of dot energies become nonlinear. The characteristic fields of crossing of the states $B_{c}$ are modified by valley mixing, for $\Delta_{K K^{\prime}}=0 B_{c}=B_{s}=0.5$ (Fig. 7a) and for $\Delta_{K K^{\prime}}=0.3 B_{c}=0.47$ (Figs. $7 \mathrm{~b}$ and $7 \mathrm{c}$ ). For strong mixing no ground state crossing is observed (inset of Fig. 7c). Field induced SU(2) Kondo effect results from the effective fluctuation of isospin characterizing Kramers doublet. The isospin in this case is neither the pure spin nor valley pseudospin, but a combination of both and its screening does not mean quenching of pure spin nor valley pseudospin. It reflects in significant and almost equal contribution of all four spin-orbital channels to total conductances, both at zero field and for the field of revival of Kondo correlations (Fig. 7a). When valley mixing is 

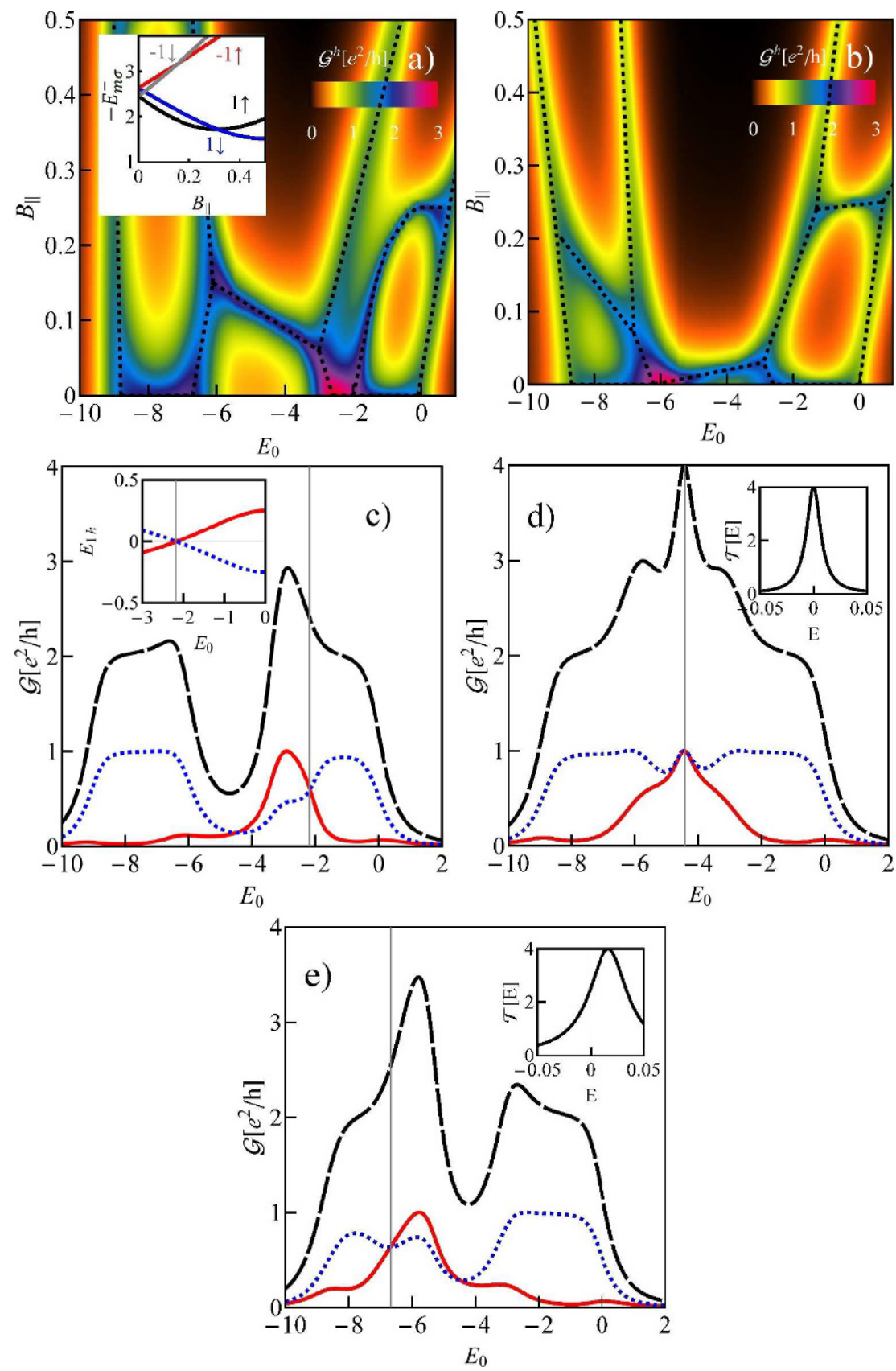

Fig. 9. Small bandgap CNTQD. (a,b) Hole conductance map $\mathcal{G}^{h}\left(E_{0}, B_{\|}\right)$for $\Delta_{g}=2$ (a) and $\Delta_{g}=5$ (b) $\left(\Delta_{Z}=-1 / 2\right.$, $\Delta_{O}=3 / 4, \mu_{o}=5$ ). Inset presents field dependencies of single-hole states. (c,d,e) Zero field conductances for $N_{h}=1, \Delta_{g}=2$ (b), $N_{h}=2, \Delta_{g}=4$ (c), $N_{h}=3, \Delta_{g}=6$ (d) (solid lines). Dotted and dashed lines present partial conductances corresponding to the spin-orbital doublets. Inset of (a) illustrates crossing of two single-hole doublets and insets of (c,d) present the corresponding transmissions of $\mathrm{SU}(4)$ Kondo resonances for $N_{h}=2$ and $N_{h}=3$.

absent and valley quantum number is preserved, only two channels are opened (Fig. 7b). To elucidate the role of spin and valley pseudospin in Kondo fluctuations we present spin, valley and Kramers polarizations of conductance for $\Delta_{K K^{\prime}}=0$ (Fig. $7 \mathrm{~d}$ ) and in the inset for finite valley mixing. For $B=0$ spin and valley polarizations vanish both for finite and zero valley mixing. Kramers polarization is finite and indicates which Kramers doublet is active in Kondo processes, for $\Delta_{K K^{\prime}} \neq 0$ pure spin-orbital Kramers polarization considerably decreases.
For the fields of Kondo revivals, spin conductance polarizations vanish, but Kondo states become valley polarized, opposite in two cases.

\subsection{Intervalley scattering induced by Coulomb interaction}

In Section 3.2 we have discussed intervalley scattering resulting from disorder on the scale of interatomic spacing and indirect valley mixing, where interference processes 


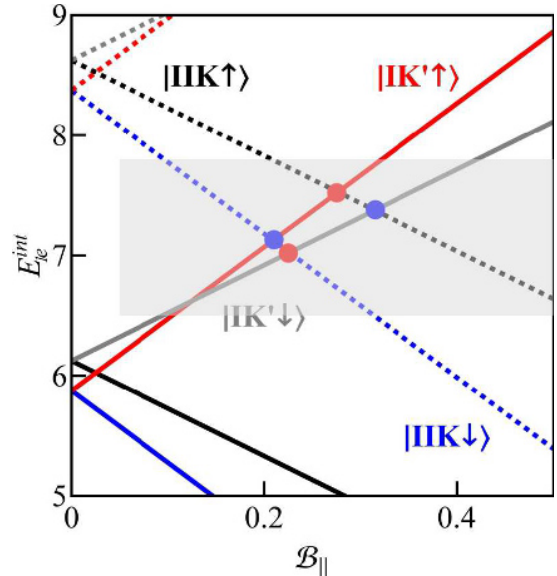

Fig. 10. Schematic diagram of energy levels of two consecutive CNTQD shells $\left(E_{1 e}^{i n t}=E_{1 e}-2 \mathcal{U}\right.$, where $E_{1 e}$ is the shifted dot energy in the lower shell). The solid lines present energies of the lower shell and the dotted lines the energies of the upper. The blue dots mark valley crossing for the same spin channel and red dots indicate spin-valley crossing.

with the electrode states played the essential role. Here we analyze mixing between valleys caused by local part of electron-electron interaction. The dominant part of Coulomb interaction - long range interaction is diagonal in valley and spin degrees of freedom. The corresponding scattering processes, called forward scattering (FS), are associated with small momentum transfer. For short quantum dots also local interactions, which can exchange isospin come into play (backward scattering term VBS [76-79]). This term is described by [78]:

$$
\mathcal{H}_{V B S}=\frac{V}{2} \sum_{\sigma \sigma^{\prime}} c_{m \sigma}^{\dagger} c_{-m \sigma^{\prime}}^{\dagger} c_{m \sigma^{\prime}} c_{-m \sigma}
$$

Isospin flip implies a momentum transfer of the order $1 / a$ $(a$ - lattice constant) and it occurs when electrons have non-negligible probability of being closer than a distance $a$. VBS term can be viewed as an effective intervalley exchange, since it lifts the degeneracy of the spin polarized and valley polarized states. The confirmation of this view was the observation of huge enhancement of effective spin-orbit splitting at half filling of hole shell [50]. A similar observation was also reported for electrons [79]. The difference between odd and even filling of the nanotube multiplets was attributed to the effective intervalley backscattering. VB scattering acts on a much smaller energy scale than FS scattering, but these processes are relevant for the problems discussed by us, because their energy is comparable with SO interaction energy and Kondo temperature. We focus on two electron case. Due to large separation of longitudinal modes, when one can restrict to a good approximation to the single longitudinal mode, the CNTQD wave function can be decomposed into a spatial and a spin-isospin components. Since the total wave function of two electrons has to be antisymmetric under exchange of electrons, the antisymmetric function in real space implies symmetric form in spin-isospin sector and vice versa. The ground state of the two-electron system has a symmetric orbital wave function with corresponding six antisymmetric spin-valley functions. They can be labeled by total helicity $\kappa=\sigma_{1} m_{1}+\sigma_{2} m_{2}$, total spin $\sigma=\sigma_{1}+\sigma_{2}$ and isospin $m=m_{1}+m_{2},(|\kappa m \sigma\rangle)$. SO interaction acts only on the states $\kappa \neq 0$, VB scattering on the states $m=0$, and total spin remains a good quantum number. As has been discussed in Section 3.1, in the absence of magnetic field SO interaction splits the sixfold multiplet into two singlets $(\kappa=\overline{2}, 2$ and quartet $\kappa=0)$. In effect of backscattering $\kappa=0$ quartet is further split by $\Delta_{V B S}$ with the corresponding lower states, which are spin polarized $\left(S_{2}=|002\rangle\right.$, $\left.S_{\overline{2}}=|00 \overline{2}\rangle\right)$ and higher states, valley polarized $\left(V_{2}=\right.$ $\left.|020\rangle, V_{\overline{2}}=|0 \overline{2} 0\rangle\right)$. The valley polarized doublet is raised with respect to spin polarized doublet. VB scattering does not conserve helicity and spin-valley unpolarized states $(\kappa=\overline{2}, 2)$ become mixed by VBS perturbation $\left(S V_{ \pm}=\right.$ $1 / \sqrt{2}(|\overline{2} 00\rangle \pm|200\rangle))$. The corresponding shifts of energies are $\pm \Lambda= \pm \sqrt{\Delta^{2}+\Delta_{V B S}^{2}}$ [78]. The values of $\Delta_{V B S}$ calculated from the observed effective SO splitting $\Lambda$ are $\Delta_{V B S}=0.2 \mathrm{meV}$ [79] and $\Delta_{V B S}=1.56 \mathrm{meV}$ [50]. Estimates from uncorrelated states based on first-principles perturbation theory predict $\Delta_{V B S}$ to be of hundreds of $\mu \mathrm{eV}[6,78]$ and other theoretical estimates give much smaller values of $\Delta_{V B S} \sim 1-10 \mu \mathrm{eV}$ [77-79]. There are suggestions that neglect of superexchange with participation of the state from different shell is responsible for an underestimation of valley exchange [6]. Since there is no clear view in literature on the relative value of VBS parameter $V$ in relation to SO splitting, and even the sign of $V$ is the subject of discussion [6] we analyze both cases $V>0$ and $V<0$. The examples of field evolution of the mentioned two-electron dot eigenstates are shown for weak VBS case $(|V / \Lambda| \ll 1)$ in Figure 8a, and in the strong VB scattering limit $(|V / \Lambda| \sim 1)$ in Figure 8b. Interesting observation shown in the inset of Figure $8 \mathrm{~b}$ is that apart from the field induced recovery of double degeneracy, for strong VBS, also triple degeneracy may appear. Figures $8 \mathrm{c}$ and $8 \mathrm{~d}$ show examples of the field dependencies of conductance in the presence of VB scattering and insets present the ground state maps of the dot with lines, which mark cross sections, for which conductances have been drawn. The results are presented for $\mathcal{U}^{\prime}<\mathcal{U}$, because no triple degeneracy point appears on the ground state map for $\mathcal{U}=\mathcal{U}^{\prime}$ for $V<0$. For $V>0$ triple degeneracy occurs also for $\mathcal{U}=\mathcal{U}^{\prime}$ (left inset of Fig. $8 \mathrm{~d})$, but the degeneracy region on the $(\Delta, B)$ map is considerably smaller than for $\mathcal{U}^{\prime}<\mathcal{U}$ (right inset). Conductance curves with two maxima reflect two consecutive field induced Kondo effects, maxima for lower fields correspond to spin $\mathrm{SU}(2)$ resonances and for higher fields spin-valley Kondo effects. Conductance curves with the single maximum correspond to $\mathrm{SU}(3)$ Kondo effect. The corresponding transmission peak of this many-body resonance of enhanced symmetry is asymmetric and is shifted away from the Fermi energy and is characterized by a phase shift $\delta \sim \pi / 3$ (lower inset of Fig. 8c). We also present partial conductances for $\mathrm{SU}(3)$ case, and it 

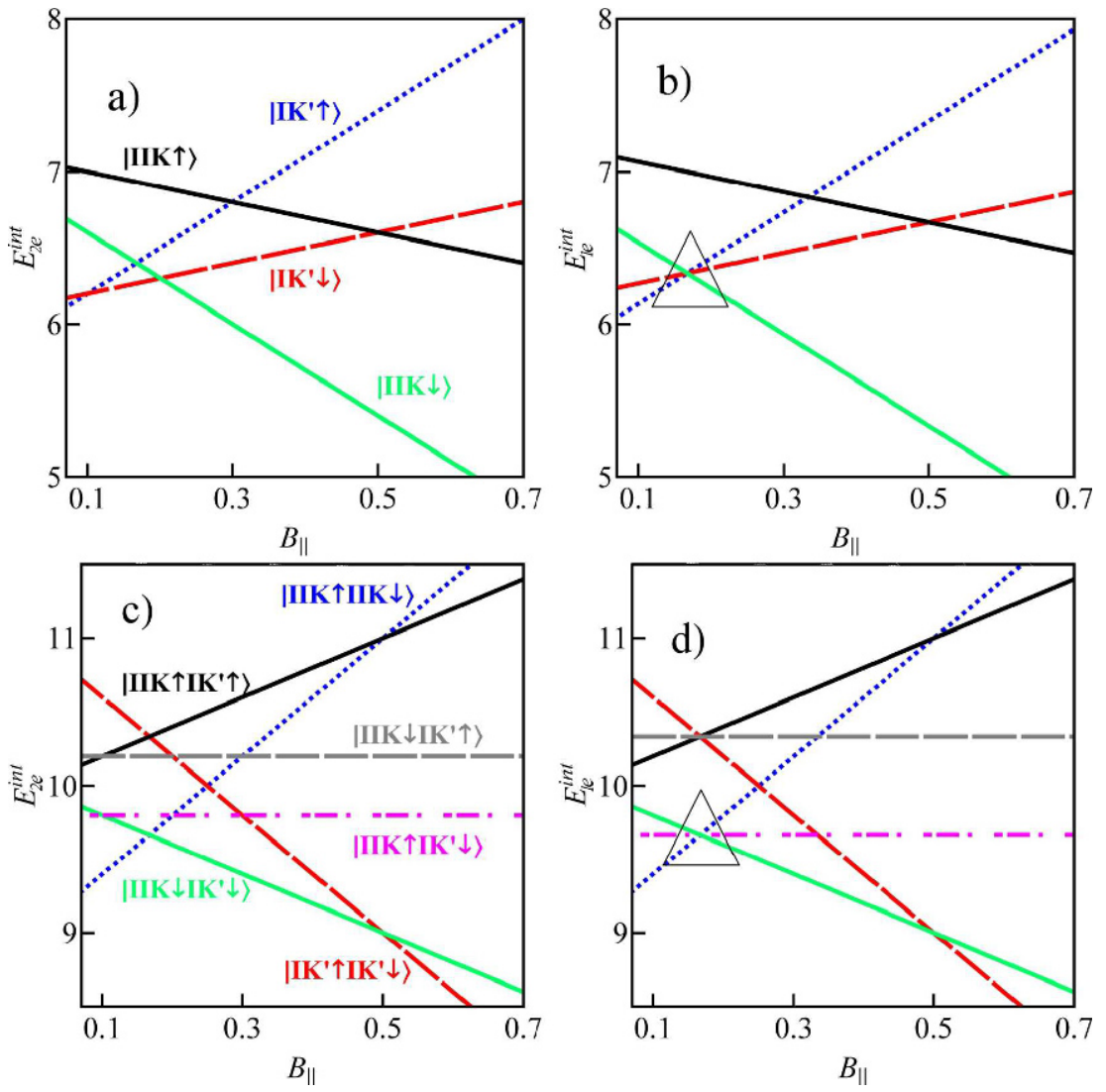

Fig. 11. Energies in intershell manifold. (a,b) Field dependencies of single-electron energies for $\Delta=1 / 10$ (a) and $\Delta=1 / 6$ (b). (c,d) Two-electron energies for $\Delta=1 / 10$ and $\Delta=1 / 6$ correspondingly $\left(E_{2 e}^{i n t}=E_{2 e}-2 \mathcal{U}\right)$. Energy separation of the shells $\Delta E=1$. Triangles on (b) and (d) mark SU(3) cases.

is seen that practically only three channels participate in transport and they equally contribute to the total conductance $\left(\mathcal{G} \sim(9 / 4)\left(e^{2} / h\right)\right)$. The residual value derived from the fourth channel only slightly enhances the total conductance.

\subsection{Small bandgap carbon nanotube quantum dots}

The small gaps occur in the nanotubes which in the simple zone folding picture should be nominally metallic. These gaps do not result from quantization of momentum along circumference (gaps of order of few hundred meV), but are induced by curvature or strain (band gaps $\leq 10 \mathrm{meV}$ ) [9]. Nearly metallic nanotubes exhibit crossing of the Dirac points at anomalously low magnetic fields $\left(B_{\text {Dirac }} \sim 2 \mathrm{~T}\right.$ $[9,80])$, what indicates the small shifts of circumference quantization lines from the Dirac point and thus confirm the small values of the bandgaps. The small gaps are comparable with the energies of SO splitting or valley mixing and therefore the commonly used large gap expansion of single electron energies cannot be applied in this case. Using the full expression (4) the parabolic field dependencies of single electron energies result (see the inset of Fig. 9a). These dependencies are determined not only by the response of orbital and spin magnetic moments, as in the case of large gaps, but crucially depend also on the value of the gap and gate voltage. Consequently neither
Coulomb lines nor the Kondo lines are straight lines on gate voltage- magnetic field conductance maps (Fig. 9a). The fields of Kondo recovery depend on voltage and this manifests in that the Kondo lines are not parallel to the gate axis. As it is seen the maps do not exhibit intrashell $e-h$ symmetry. Apart from $2 e$ valley, recovery of Kondo effect is observed not only in one, but in both odd valleys (Fig. 9b). This is in contrast to what is observed in wide gap nanotubes, where depending on the sign of SO coupling Kondo effect appears in $1 e$ or $3 e$ valley (Sect. 3.3). The ability to restore degeneration in both odd valleys is again a consequence of nonlinear field dependence of energies. The surprising result is observed also at zero magnetic field. For some values of the gap, different for different SO couplings, Kondo effect of SU(4) symmetry can appear. Due to nonlinear gate dependence of the dot energies characteristic for small gap carbon nanotubes, the effective spin-orbit splitting tends to zero for some values of the bandgap and SO parameters. The example for $N=1$ is presented in the inset of Figure 9c. For a certain value of gate voltage the curves cross, what means vanishing of the effective spin-orbit splitting and SU(4) symmetry is recovered. Similar crossings of the states, for different values of SO coupling are also possible in $N=2$ and $N=3$ valleys. Figures $9 \mathrm{c}$ and $9 \mathrm{e}$ illustrate, how crossover to Kondo effect of higher symmetry in odd valleys $(\mathrm{SU}(2) \rightarrow \mathrm{SU}(4))$ manifest in conductance and 

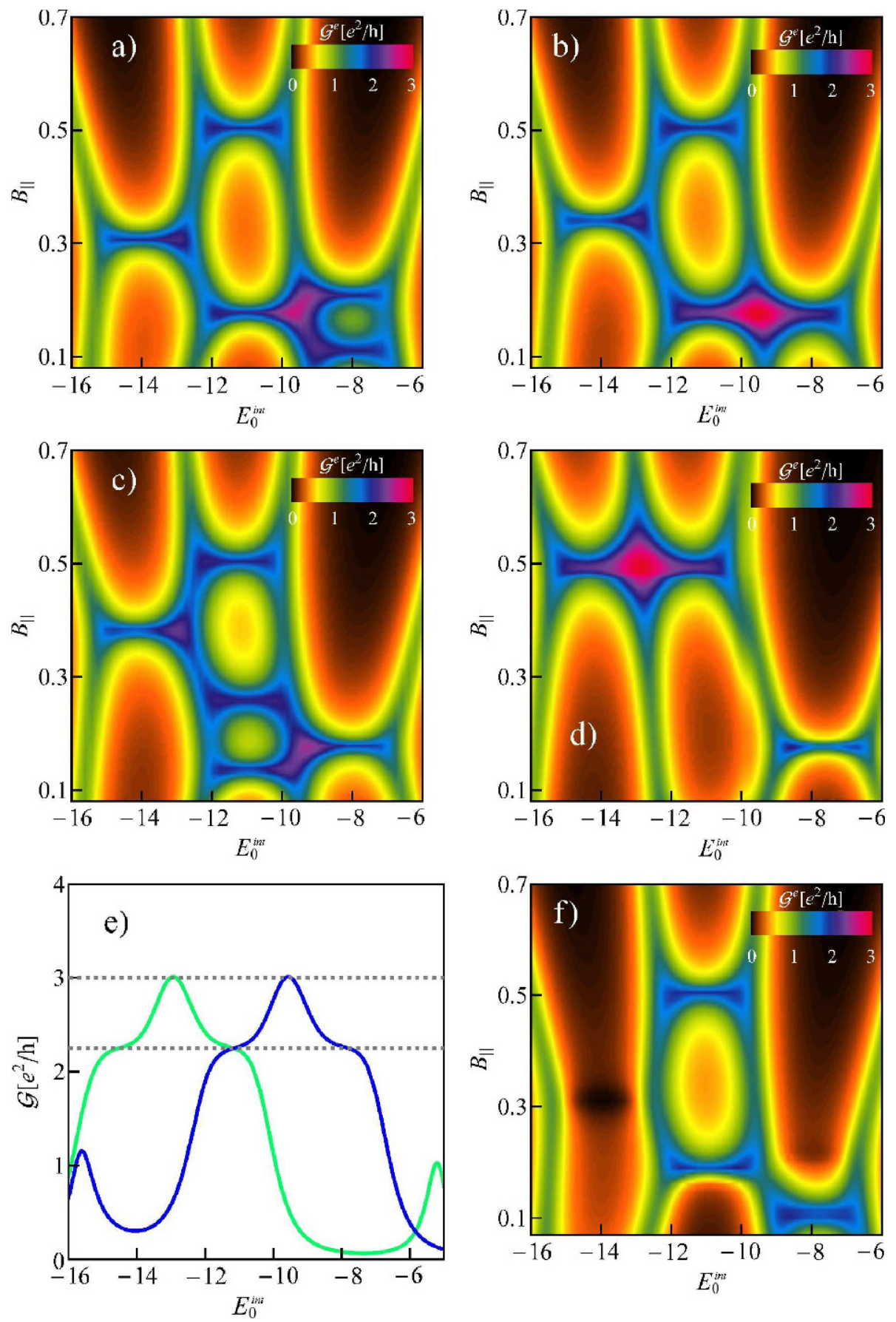

Fig. 12. Intershell gate-magnetic field conductance maps $\left(E_{0}^{\text {int }}=E_{0}-2 \mathcal{U}\right)$. (a) $\Delta=1 / 10,(\mathrm{~b}), \Delta=1 / 6$ (SU(3) Kondo for $N=1,2)$ (c), $\Delta=1 / 4$, (d) $\Delta=1 / 2$ (SU(3) Kondo for $N=2,3$ ). (e) Conductances presented for magnetic fields, for which $\mathrm{SU}$ (3) Kondo effects occur $\Delta=1 / 6, B_{\|}=1 / 6$ (broken line), $\Delta=1 / 2, B_{\|}=1 / 2$ (solid). (f) Conductance for $\Delta=1 / 10$, with finite intershell intervalley mixing $\Delta_{K K^{\prime}}^{\text {int }}=1 / 10$.

similarly Figure 9d presents response of conductance on the rebuild of Kondo correlations destroyed by $\mathrm{SO}$ coupling in $N=2$ region. It is hard to keep track of the crossover in odd valleys observing only the evolution of total conductance with the change of the gap, because linear conductance for $\mathrm{SU}(4)$ symmetry coincides with that for $\mathrm{SU}(2)$, but $\mathrm{SU}(4)$ Kondo effect reflects in equal values of partial spin-orbital conductances reaching values of
$1 / 2\left(e^{2} / h\right)$ each (Figs. 9c and 9e). In even valley the gate induced rebuild of $\mathrm{SU}(4)$ Kondo correlations manifests not only in partial conductances, which have equal values $e^{2} / h$, but in this case it reflects also in total conductance by the emergence of a distinct peak. The described phenomena allow switching between transport Kondo regimes of different symmetries or even between Kondo and nonKondo states by small changes of gate voltage. Having 
in mind the property that strain can modify the gap in nearly metallic nanotubes [81] and thus can in some cases induce Kondo correlations in $2 e$ valley and opens new transport channels, one can think about possibility to use this mechanism for nano-mechanical switching of the current.

\section{Intershell Kondo effects}

The electron energies of CNTQD are quantized by periodic boundary conditions along the circumference (quantization of $k_{\perp}$ ) and by longitudinal confinement (quantization of $k_{\|}$). For small diameter the level spacing corresponding to the circumference quantization is large, of order of $0.5-1 \mathrm{eV}$ [82], and it is enough to restrict, similarly as we have done so far, to a single pair of clockwise and anticlockwise modes. In this approximation the only quantum number reflecting circumferential quantization is valley pseudospin. In most cases a similar argument on large level spacing applies also to longitudinal modes. In general, the energies of the longitudinal modes depend on the bandgap and on the details of confining potential. In the case of a sharp confining potential and for states lying not to close to the gap, the resulting quantized energies are inversely proportional to the dot length. Typically they are of order of several meV [83] and usually these energies are much larger than the rest of energies relevant for the issues discussed here. Restriction to a single shell i.e. to a manifold of four states $(|K \sigma\rangle)$ corresponding to a single value of $k_{\|}$is then justified. All our previous discussion was limited to this case. In this section we abandon this assumption and focus on the intershell effects, that are relevant for higher magnetic fields or for longer dots. Figure 10 schematically illustrates spectrum of two neighboring shells vs parallel magnetic field. In the following we concentrate on the description of many-body processes only for the high fields, where intershell crossings of the states occur and the states from the lower doublet of the lower shell and similarly the states form higher doublet of the higher shell are well separated from the rest and with a good approximation it is enough to restrict to the intershell manifold (IM) of the states $\left(|I M\rangle=\mid I K^{\prime} \downarrow\right.$ \rangle$,\left|I K^{\prime} \uparrow\right\rangle,|I I K \downarrow\rangle,|I I K \uparrow\rangle$ ) (the first quantum numbers label the shells). In other words, instead of analyzing more generally all occupation regions from both shells, we focus on the high field regions of $N_{I}=3,4$ occupations of lower shell and $N_{I I}=1,2$ for higher shell. Alternatively we can look at this region as single occupied range of (IM) $N_{\text {int }}=1\left(N_{I}=3\right)$, and similarly double occupied $N_{\text {int }}=2\left(N_{I}=4\right)$, triple occupied $N_{i n t}=3\left(N_{I I}=1\right)$ and full occupied $N_{\text {int }}=4\left(N_{I I}=2\right)$. The considered region of energies and fields is shown in Figure 10 (box bounded by dotted lines). Formally the many body correlations can be described within SB formalism, similarly to the cases discussed in the previous paragraphs, by 16 slave bosons. There are two different types of intershell line crossings: spin conserving crossing (valley $\mathrm{SU}(2)$ ) or crossing of the lines of opposite spins (spin-valley SU(2)). Figure 12 shows conductance maps for several values of spin-orbit splitting. The number of Kondo enhanced conductance lines appearing in each occupancy region and the characteristic fields, for which the lines appear, depend on the value of $\Delta$ and they correspond to degeneracy recovery in the presented field range. As an example let us analyze conductance for $\Delta=1 / 10$ (Fig. 12a). The lower line for $N_{\text {int }}=1\left(E_{0}^{\text {int }} \sim-7.5\right)$ is the intershell Kondo resonance $\left(B_{\|}=0.1\right)$ and the higher at $B_{\|}=0.2$ reflects Kondo revival due to cotunneling induced effective intershell valley quantum fluctuations between the states $\left|I K^{\prime} \downarrow\right\rangle$ and $|I I K \downarrow\rangle$ (see Fig. 11a). In $N_{\text {int }}=3$ region $\left(E_{0}^{\text {int }}=-13.5\right)$ intershell valley Kondo effect occurs at $B_{\|}=0.3$ due to effective fluctuations between the $|I I K \uparrow\rangle$ and $\left|I K^{\prime} \uparrow\right\rangle$ states (not presented). In both cases significant conductance spin polarization is observed (Figs. 12a and 13c), because Kondo processes occur within the single spin channels (for $N_{\text {int }}=1 P C_{s} \approx-1$ and for $N_{\text {int }}=3 P C_{s} \approx$ 1). In $N_{\text {int }}=2$ region two Kondo lines correspond to intershell spin-valley effects, the lower occurring for $B_{\|}=1 / 6$ results from $|I I K \uparrow I I K \downarrow\rangle$ and $\left|I I K \downarrow I K^{\prime} \downarrow\right\rangle$ fluctuations and the higher for $B_{\|}=1 / 2$ from $|I I K \uparrow I I K \downarrow\rangle$ and $\left|I K^{\prime} \uparrow I K^{\prime} \downarrow\right\rangle$ fluctuations (Fig. 11c). In both cases the corresponding conductances are unpolarized (Figs. 12a and 13c). Apart from the field induced SU(2) Kondo revivals also higher symmetry effects are possible. For the assumed parameters it holds for $\Delta=1 / 6$ and $\Delta=1 / 2$. As it is illustrated for $\Delta=1 / 6$ in Figure $11 \mathrm{~b}$ (single electron states) and Figure 11d (two-electron states) magnetic field brings three states to degeneracy. Each of these states is coupled with equal strength to the corresponding state in electrode and in the considered strong coupling limit Kondo $\mathrm{SU}(3)$ effect appears at half filling $(N=2)$ and in one of odd occupied regions $(N=3$ for $\Delta=1 / 6$, or $N=1$ for $\Delta=1 / 2$ ). It manifests by the enhanced conductance, which in the limit of small coupling to the electrodes approaches $\mathcal{G}=(9 / 4)\left(e^{2} / h\right)$ value. The conductance plotted for the fields, for which the threefold degeneracy occurs exhibit clear plateaus evidencing the appearance of the mentioned Kondo effects (Fig. 12e). Let us close this section by a short remark on the impact of intershell intervalley mixing. Figure $12 \mathrm{f}$ shows conductance map for $\Delta_{K K^{\prime}}^{i n t}=0.1$, corresponding map for $\Delta_{K K^{\prime}}^{i n t}=0$ is presented in Figure 12a. In the $N=3$ region, instead of Kondo enhanced line, line of strongly reduced conductance is observed for finite valley mixing and in region of single occupancy instead of two Kondo lines only one broadened line is seen. To understand these changes we show in Figures 13a and 13b the evolution of conductances with increasing valley mixing drawn for the gates corresponding to the centers of $N_{\text {int }}=3$ and $N_{\text {int }}=1$ areas. We additionally present in the insets schematic views of the field dependencies of pure dot electron states perturbed by valley mixing. For $N_{i n t}=3$ anticrossing of the ground state with one of the states from the higher shell occurs and consequently the Kondo peak splits and conductance maximum first lowers for small $\Delta_{K K^{\prime}}^{\text {int }}$ and for stronger valley mixing a dip is observed. For $N_{i n t}=1$ a similar anticrossing of the states from different shells is observed with correspondingly reduced conductance $\left(B_{\|}=0.2\right)$, but for slightly lower field $\left(B_{\|}=0.1\right)$ crossing 

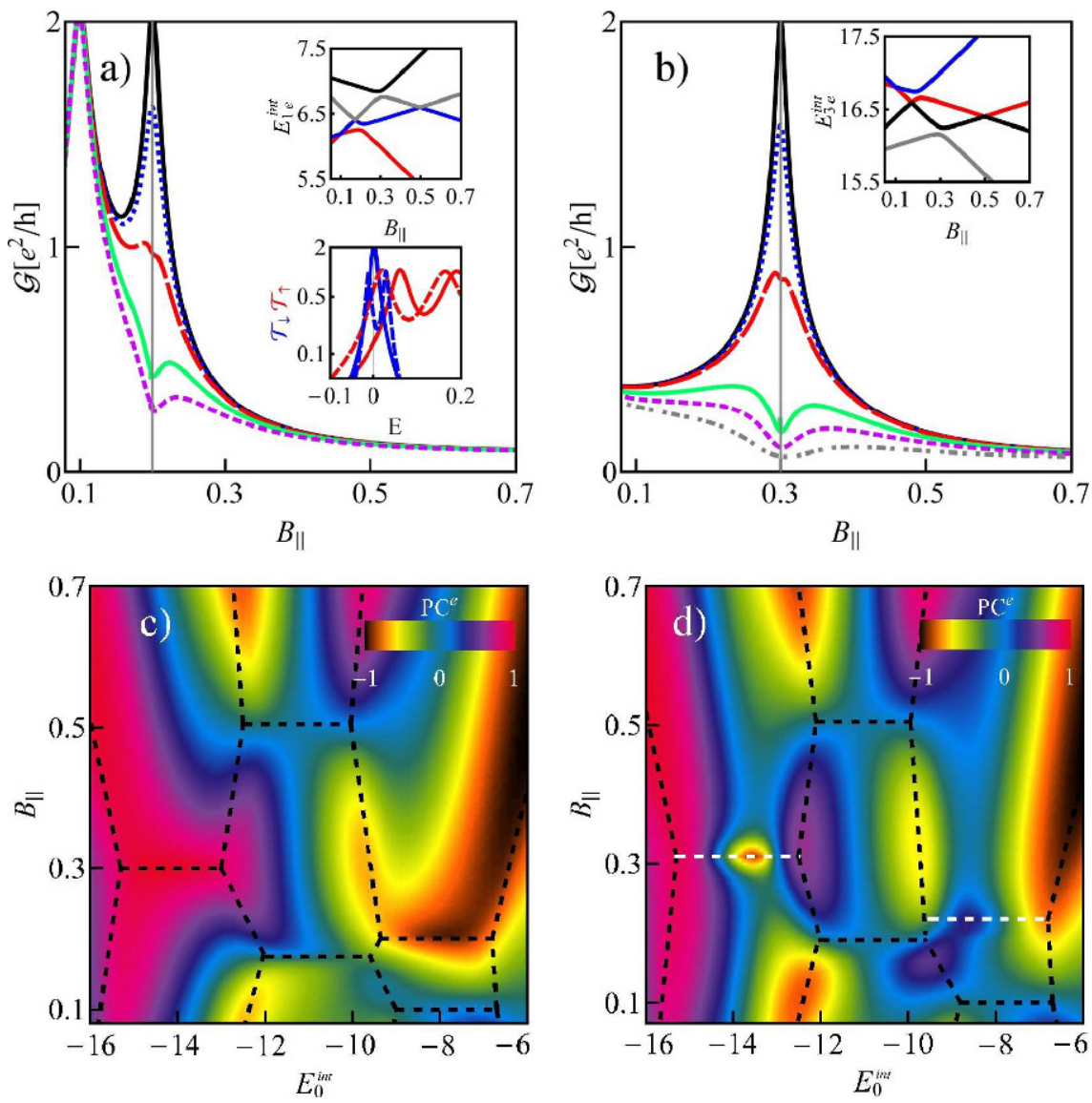

Fig. 13. Conductances for $\Delta=0.1$ presented for (a) the centre of intershell occupation region $N=1\left(E_{0}^{\text {int }}=-7.5\right)$ and (b) centre of intershell occupation $N=3\left(E_{0}^{\text {int }}=-13.5\right)$ for different intershell valley mixings: $\Delta_{K K^{\prime}}^{\text {int }}=0$ (solid black line), $\Delta_{K K^{\prime}}^{i n t}=0.005$ (dotted blue), $\Delta_{K K^{\prime}}^{i n t}=0.01$ (dashed red), $\Delta_{K K^{\prime}}^{i n t}=0.02$ (green) and $\Delta_{K K^{\prime}}^{i n t}=0.03$ (short dashed magenta). Insets shows the field evolutions of single- electron energies (a) and energies of three-electron states of intershell manifold (b) $\left(E_{3 e}^{i n t}=E_{3 e}-2 \mathcal{U}\right)$. Spin polarization map of conductance for $\Delta=0.1$ and $\Delta_{K K^{\prime}}^{\text {int }}=0$ (solid black line), $\Delta_{K K^{\prime}}^{\text {int }}=0.005$ (dotted blue), $\Delta_{K K^{\prime}}^{i n t}=0.01$ (dashed red), $\Delta_{K K^{\prime}}^{i n t}=0.02$ (green), $\Delta_{K K^{\prime}}^{i n t}=0.03$ (short dashed magenta) and $\Delta_{K K^{\prime}}^{i n t}=0.05($ dashed dotted gray). (c,d) Spin polarization map of conductance for $\Delta=0.1, \Delta_{K K^{\prime}}^{i n t}=0$ and $\Delta_{K K^{\prime}}^{i n t}=0.01$.

of the states from the same (lower) shell occurs leading to a modified Kondo line. The latter problem of restoring of the Kondo resonance within single shell has been already discussed in Section 3.3. Comparing the spin polarizations of conductance presented in Figure $13 \mathrm{c}$ for $\Delta_{K K^{\prime}}^{i n t}=0$ and $\Delta_{K K^{\prime}}^{i n t} \neq 0$ (Fig. $13 \mathrm{~d}$ ) it is seen that the valley mixing induced splitting of the Kondo resonance results in the change of sign of polarization for $N_{\text {int }}=1,3$. To get insight into this effect we present in the lower inset of Figure 13 a example for transmission for $N_{\text {int }}=1$. $\mathcal{T}_{\downarrow}$ represents Kondo resonance, which for $\Delta_{K K^{\prime}}=0$ is an unsplit peak (compare Fig. 11a), whereas $\mathcal{T}_{\uparrow}$ reflects excited processes involving spin up states lying higher in energy and this line is split already for vanishing valley mixing. For $\Delta_{K K^{\prime}} \neq 0$ the energies of Kondo active states differ and instead of location of the Fermi level at the peak of $\mathcal{T}_{\downarrow}\left(\Delta_{K K^{\prime}}=0\right)$, for finite mixing $E_{F}$ places close to the $\operatorname{dip} . \mathcal{T}_{\uparrow}$ shifts towards lower energies with the increase of $\Delta_{K K^{\prime}}$ and continues to split. For sufficiently large valley mixing $\mathcal{T}_{\downarrow}$ dominates at the Fermi level and reverse of polarization results.

\section{Concluding remarks}

We have studied the interplay of different symmetry breaking perturbations on transport through CNTQD in the range of strong correlations. Spin-orbit interaction or valley mixing breaks the spin-orbital symmetry in carbon nanotubes and in odd occupied valleys lifts the fourfold degeneracy of the states leaving Kramers double degeneracy. A crossover from highly symmetric SU(4) Kondo effect into lower symmetry SU(2) then results. At half filling the sixfold degeneracy is removed under these perturbations and a quartet and two singlets appear. For strong perturbation Kondo correlations are destroyed in this case, because the dot state of the lowest energy is singlet. Magnetic field breaks time-reversal symmetry and degeneracy is completely lifted and consequently Kondo resonances disappear for fields exceeding Kondo energy scale. Magnetic field acts both on spin and orbital (valley) pseudospin, in the case of wide gap nanotubes field linearly increases or decreases the energies depending on the orientations of magnetic moments associated with 
the dot states. At some fields the degeneracy of some states might be recovered and revival of Kondo effect can occur. Depending which states come to degeneracy, the screened isospin in Kondo processes is a pure spin or orbital pseudospin or a mixture of both, what reflects in complete or only partial vanishing of spin or valley polarizations. By applying gate voltage one can change the region of the dot occupations, but for wide gap tubes it is not possible electrically recover the degeneracy within the same occupation range. In nearly metallic nanotubes the field evolution of state energies is not linear and then not only magnetic field, but also gate voltage can recover degeneracy within a valley of a given occupation, opening the conditions for building up of Kondo correlations. Interesting, in these narrow gap systems the gate induced reconstruction of dot states can compensate in specific conditions the changes induced by spin-orbit coupling and revival of $\mathrm{SU}(4)$ Kondo effect can result at zero magnetic field. Kondo physics in CNTQDs is even richer if one includes the impact of other perturbations e.g. local Coulomb interaction induced valley back scattering, important for short QDs, or intershell effects, which may play the role for longer dots and in high magnetic fields. In these cases the possibility of the occurrence of $\mathrm{SU}(3)$ Kondo effect is foreseen by us. To get more comprehensive description of intra- and intershell many-body effects on equal footing extended basis of states from both shells have to be considered requiring a use of more slave boson operators. This problem will be discussed elsewhere [84]. Also the presented discussion of SU(3) Kondo effect is only preliminarily, the more detailed analysis is under our investigation. Theoretical challenge which we encounter considering many-body processes in the presence of VBS perturbation is the impact of VBS induced intradot entanglement of the dot states on the cotunneling processes in which participate also unentangled states of electrodes. Even in the case of the full geometrical symmetry, the mentioned distinction of electrode and dot states reflects in Kondo resonance. Zero frequency transmissions of the highly symmetric many-body resonances $(\mathrm{SU}(\mathrm{N}))$ are identical for the degenerate states, but as suggested by our preliminary results they slightly differ (depending on the degree of entanglement) for finite frequencies. This differentiates this case from the situation when only product dot and electrode states participate in many-body processes, then all $\mathrm{N}$ partial transmissions are identical. It is also worth pointing out that taking into account states from more than two shells degeneracy of higher order than three can occur at high fields. Rapid progress in technology allows production of ultraclean CNTQDs making them the ideal objects for studying different strongly correlated regimes. The richness of many-body states occurring in these systems results from a competition of different interactions and from different degrees of freedom involved in formation of resonances in the strongly correlated CNTQDs. This is promising for information processing, because it opens the path for magnetic, electric or mechanical manipulation not only of the spin, but also valley degree of freedom, or both of them.

\section{Author contribution statement}

Both authors contributed equally to the paper.

Open Access This is an open access article distributed under the terms of the Creative Commons Attribution License (http://creativecommons.org/licenses/by/4.0), which permits unrestricted use, distribution, and reproduction in any medium, provided the original work is properly cited.

\section{References}

1. R. Saito, G. Dresselhaus, M.S. Dresselhaus, Physical properties of carbon nanotubes (Imperial College Press, UK, 1998)

2. A. Cottet, T. Kontos, S. Sahoo, H.T. Man, M.-S. Choi, W. Belzig, C. Bruder, A.F. Morpurgo, C. Schönenberger, Semicond. Sci. Technol. 21, S78 (2006)

3. A. Javey, J. Kong (eds.) Carbon nanotube electronics (Springer, Boston, 2009)

4. P. Avouris, Phys. Today 62, 34 (2009)

5. M. Shulaker, G. Hills, N. Patil, H. Wei, H.-Y. Chen, H.-S.P. Wong, S. Mitra, Nature 501, 526 (2013)

6. E.A. Laird, F. Kuemmeth, G. Steele, K. Grove-Rasmussen, J. Nygård, K. Flensberg, L.P. Kouwenhoven, Rev. Mod. Phys. 87, 703 (2015)

7. N. Hamada, S.I. Sawada, A. Oshiyama, Phys. Rev. Lett. 68, 1579 (1992)

8. R. Saito, M. Fujita, G. Dresselhaus, Appl. Phys. Lett. 60, 2204 (1992)

9. G.A. Steele, F. Pei, E.M. Larid, J.M. Jol, H.B. Meerwaldt, L.P. Kouvenhoven, Nat. Commun. 4, 1573 (2013)

10. J. Cao, Q. Wang, H.J. Dai, Nat. Mater. 4, 745 (2005)

11. F. Kuemmeth, S. Ilani, D.C. Ralph, P.L. McEuen, Nature 452, 448 (2008)

12. E.D. Minot, Y. Yaish, V. Sazanova, P.L. McEuen, Nature 428, 536 (2004)

13. D.H. Cobden, J. Nygård, Phys. Rev. Lett. 89, 046803 (2002)

14. P. Jarillo-Herrero, J. Kong, H.S.J. Van der Zant, C. Dekker, L.P. Kouvenhoven, S. De Franceschi, Nature 434, 484 (2005)

15. A. Makarovski, J. Liu, G. Finkelstein, Phys. Rev. Lett. 99, 066801 (2007)

16. K. Grove-Rasmussen, H.J. Jorgensen, P.E. Lindelof, Physica E 40, 92 (2007)

17. F. Wu, R. Danneau, P. Queipo, E. Kauppinen, T. Tsuneta, P.J. Hakonen, Phys. Rev. B 79, 073404 (2009)

18. M. Ferrier, T. Arakawa, T. Hata, R. Fujiwara, R. Delagrange, R. Weil, R. Deblock, R. Sakano, A. Oguri, K. Kobayashi, Nat. Phys. 12, 230 (2016)

19. M. Ferrier, T. Arakawa, T. Hata, R. Fujiwara, R. Delagrange, R. Weil, R. Deblock, Y. Teratani, R. Sakano, A. Oguri, K. Kobayashi, Phys. Rev. Lett. 118, 196803 (2017)

20. T. Delattre, C. Feuillet-Palma, L.G. Herrmann, P. Morfin, J.-M. Berroir, G. Fève, B. Plaçais, D.C. Glattli, M.-S. Choi, C. Mora, T. Kontos, Nat. Phys. 5, 208 (2009)

21. T. Pohjola, H. Schoeller, G. Schön, Europhys. Lett. 54, $241(2001)$ 
22. L. Borda, G. Zaránd, W. Hofstetter, B.I. Halperin, J. von Delft, Phys. Rev. Lett. 90, 026602 (2003)

23. A.I. Chudnovskiy, Europhys. Lett. 71, 672 (2005)

24. M.-S. Choi, R. López, R. Aguado, Phys. Rev. Lett. 95, 067204 (2005)

25. S. Lipiński, D. Krychowski, Phys. Stat. Sol. (b) 243, 206 (2006)

26. S. Lipiński, D. Krychowski, J. Alloys Compd. 423, 215 (2006)

27. R. López, D. Sanchez, M. Lee, M.-S. Choi, P. Simon, K. Le Hur, Phys. Rev. B 71, 115312 (2005)

28. J.S. Lim, M.-S. Choi, M.Y. Choi, R. López, R. Aguado, Phys. Rev. B 74, 205119 (2006)

29. G. Zaránd, Philos. Mag. 86, 2043 (2006)

30. M.R. Galpin, D.E. Logan, H.R. Krishnamurthy, J. Phys.: Condens. Matter 18, 6571 (2006)

31. R. Sakano, N. Kawakami, Phys. Rev. B 73, 155332 (2006)

32. J. Mravlje, A. Ramšak, T. Rejec, Phys. Rev. B 73, 241305R (2006)

33. K. Le Hur, P. Simon, D. Loss, Phys. Rev. B 75, 035332 (2007)

34. C.A. Büsser, G.B. Martins, Phys. Rev. B 75, 045406 (2007)

35. T.-F. Fang, W. Zuo, H.-G. Luo, Phys. Rev. Lett. 101, 246805 (2008)

36. F.B. Anders, D.E. Logan, M.R. Galpin, G. Finkelstein, Phys. Rev. Lett. 100, 086809 (2008)

37. S. Lipiński, D. Krychowski, Phys. Rev. B 81, 115327 (2010)

38. A Makarovski, A. Zhukov, J. Liu, G. Finkelstein, Phys. Rev. B 75, 241407 (2007)

39. A.J. Keller, S. Amasha, I. Weymann, I.G. Rau, J.A. Katine, H. Shtrikman, G. Zarand, D. Goldhaber-Gordon, Nat. Phys. 10, 145 (2013)

40. M. Filippone, C.P. Moca, G. Zaránd, C. Mora, Phys. Rev B 90, 121406 (2014)

41. D.R. Schmid, S. Smirnov, M. Margańska, A. Dirnaichner, P.L. Stiller, M. Grifoni, A.K. Hüttel, C. Strunk, Phys. Rev. B 91, 155435 (2015)

42. D. Krychowski, S. Lipiński, Phys. Rev. B 93, 075416 (2016)

43. T.S. Jespersen, K. Grove-Rasmussen, J. Paaske, K. Muraki, T. Fujisawa, J. Nygård, K. Flensberg, Nat. Phys. 7, 348 (2011)

44. K. Grove-Rasmussen, S. Grap, J. Paaske, K. Flensberg, S. Andergassen, V. Meden, H.I. Jorgensen, K. Muraki, T. Fujisawa, Phys. Rev. Lett. 108, 176802 (2012)

45. H.O.H. Churchill, F. Kuemmeth, J.W. Harlow, A.J. Bestwick, E.I. Rashba, K. Flensberg, C.H. Stwertka, T. Taychatanapat, S.K. Watson, C.M. Marcus, Phys. Rev. Lett. 102, 166802 (2009)

46. D.V. Bulaev, B. Trauzettel, D. Loss, Phys. Rev. B 77, 235301 (2008)

47. K. Flensberg, C.M. Marcus, Phys. Rev. B 81, 195418 (2010)

48. S.H. Jhang, M. Marganska, Y. Skourski, D. Preusche, B. Witkamp, M. Grifoni, H. van der Zant, J. Wosnitza, C. Strunk, Phys. Rev. B 82, 041404(R) (2010)

49. F. Pei, E.A. Larid, G.A. Steele, L. Kouvenhoven, Nat. Nanotechnol. 7, 630 (2012)

50. J.P. Cleuziou, N.V. N'Guyen, S. Florens, W. Wernsdorfer, Phys. Rev. Lett. 111, 136803 (2013)
51. M. Niklas, S. Smirnov, D. Mantelli, M. Margańska, N.-V. Nguyen, W. Wernsdorfer, J.-P. Cleuziou, M. Grifoni, Nat. Commun. 7, 12442 (2016)

52. T. Ando, J. Phys. Soc. Jpn. 69, 1757 (2000)

53. D. Huertas-Hernando, F. Guinea, A. Brataas, Phys. Rev. B 74, 155426 (2006)

54. W. Izumida, K. Sato, R. Saito, J. Phys. Soc. Jpn. 78, 074707 (2009)

55. J.S. Jeong, H.W. Lee, Phys. Rev. B 80, 075409 (2009)

56. T.F. Fang, W. Zuo, H.G. Luo, Phys. Rev. Lett. 104, 169902(E) (2010)

57. M.R. Galpin, F.W. Jayatilaka, D.E. Logan, F.B. Anders, Phys. Rev. B 81, 075437 (2010)

58. D. Mantelli, C.P. Moca, G. Zarand, M. Grifoni, Physica E 77, 180 (2016)

59. A. Carmi, Y. Oreg, M. Berkooz, Phys. Rev. Lett. 106 , $106401(2011)$

60. C.P. Moca, A. Alex, J. von Delft, G. Zaránd, Phys. Rev. B 86, 195128 (2012)

61. R. López, T. Rejec, J. Martinek, R. Zitko, Phys. Rev. B 87, 035135 (2013)

62. G. Kotliar, A.E. Ruckenstein, Phys. Rev. Lett. 57, 1362 (1986)

63. B. Dong, X.L. Lei, J. Phys.: Condens. Matter 13, 9245 (2001)

64. B. Dong, X.L. Lei, Phys. Rev. B 63, 235306 (2001)

65. B.R. Bułka, S. Lipiński, Phys. Rev 67, 024404 (2003)

66. P. Trocha, Phys. Rev. B 82, 125323 (2010)

67. P. Jarillo-Herrero, S. Sapmaz, C. Dekker, L.P. Kouvenhoven, H.S.J. Van der Zant, Nature 429, 389 (2004)

68. B. Babic, T. Kontos, C. Schönenberger, Phys. Rev. B 70, 235419 (2004)

69. L.P. Kouwenhoven, C.M. Marcus, P.L. McEuen, S. Tarucha, R.M. Westerwelt, N.S. Wingreen, in Proceedings of advanced study institute on mesoscopic electron transport, edited by L. Sohn, L.P. Kouvenhoven, G. Schön (Kluwer, Dordrecht, 1997)

70. P. Vitushinsky, A.A. Clerk, K. Le Hur, Phys. Rev. Lett. 100, 036603 (2008)

71. C. Mora, X. Leyronas, N. Regneult, Phys. Rev. Lett. 100, 036604 (2008)

72. A.C. Hewson, The Kondo problem to heavy fermions (Cambridge University Press, Cambridge, U.K., 1997)

73. T. Kubo, Y. Tokura, T. Hatano, S. Tarucha, Phys. Rev. B 74, 205310 (2006)

74. Ł. Karwacki, P. Trocha, Phys. Rev. B 94, 085418 (2016)

75. A. Makarovski, J. Liu, G. Finkelstein, Phys. Rev. Lett. 99, 066801 (2007)

76. T. Ando, J. Phys. Soc. Jpn. 75, 024707 (2006)

77. B. Wunsch, Phys. Rev. B 79, 235408 (2009)

78. A. Secchi, M. Rontani, Phys. Rev. B 88, 125403 (2013)

79. S. Pecker, F. Kuemmeth, A. Secchi, M. Rotani, D.C. Ralph, P.L. McEuen, S. Ilani, Nat. Phys. 9, 576 (2013)

80. W. Deshpande, B. Chandra, R. Caldwell, D.S. Novikov, J. Hone, M. Bockrath, Science 323, 106 (2009)

81. E.D. Minot, Y. Yaish, V. Sazonova, J.-Y. Park, M. Brink, P.L. McEuen, Phys. Rev. Lett. 90, 156401 (2003)

82. C. Dekker, Phys. Today 52, 22 (1999)

83. S. Reich, C. Thomsen, J. Menltzsch, Carbon nanotube: basic concepts and physical properties (Wiley, New York, 2004)

84. P. Florków, D. Krychowski, S. Lipiński (2017), to be published 